\title{
Single-particle dynamics of microbunching
}

\author{
X. J. Deng $\odot,{ }^{1,2, *}$ A. W. Chao, ${ }^{3,4}$ J. Feikes $\odot,{ }^{5}$ W. H. Huang,,${ }^{1,2 \dagger}$ M. Ries $\odot,{ }^{5}$ and C. X. Tang ${ }^{1,2}$ \\ ${ }^{1}$ Department of Engineering Physics, Tsinghua University, Beijing 100084, China \\ ${ }^{2}$ Key Laboratory of Particle and Radiation Imaging, Tsinghua University, \\ Ministry of Education, Beijing 100084, China \\ ${ }^{3}$ Institute for Advanced Study, Tsinghua University, Beijing 100084, China \\ ${ }^{4}$ SLAC National Accelerator Laboratory, Stanford University, Stanford, California 94309, USA \\ ${ }^{5}$ Helmholtz-Zentrum Berlin (HZB), Albert-Einstein-Straße 15, 12489 Berlin, Germany
}

(Received 14 October 2019; accepted 15 April 2020; published 30 April 2020)

\begin{abstract}
The mechanism of steady-state microbunching (SSMB) has been proposed [D. F. Ratner and A. W. Chao, Phys. Rev. Lett. 105, 154801 (2010)] to generate high-power coherent radiation at a high repetition rate or in continuous-wave mode using electron storage rings. In this paper, the related single-particle dynamics are theoretically and numerically studied, and important results are presented. The investigated effects are longitudinal quantum radiation excitation, nonlinear momentum compaction, and linear and nonlinear coupling of the transverse and longitudinal motion. Although this analysis is oriented toward SSMB, some of the analyzed effects are also crucial in cases such as coherent harmonic generation, bunch slicing, bunch compression, free-electron laser beam transport lines, and quasi-isochronous rings, which involve precise longitudinal phase-space manipulations.
\end{abstract}

DOI: $10.1103 /$ PhysRevAccelBeams.23.044002

\section{INTRODUCTION}

Microbunching has been one of the research focuses in accelerator physics and is expected to remain so in the years to come, as evidenced by the advent of free-electron lasers (FELs) and subsequent related developments [1-3]. When an electron beam becomes microbunched, it radiates coherently and strongly at wavelengths longer than the microbunch, amplifying the radiation intensity by orders of magnitude compared to the usual incoherent radiation. In addition to FELs, which are usually linac-based, there are also other schemes, such as coherent harmonic generation (CHG) [4-6], bunch slicing [7-9], and steady-state microbunching (SSMB) [10-12], that aim to produce either single-pass or long-term microbunching in storage rings to fulfill the various needs of users. While the great power of microbunching originates from the collective radiation effect, it seems that a systematic investigation of the related single-particle dynamics of the formation, transportation and maintenance of microstructures in linacs and storage rings is also necessary and will be of considerable value to allow greater benefits to be obtained from microbunching.

\footnotetext{
*dxj11@tsinghua.org.cn

huangwh@mail.tsinghua.edu.cn
}

Published by the American Physical Society under the terms of the Creative Commons Attribution 4.0 International license. Further distribution of this work must maintain attribution to the author(s) and the published article's title, journal citation, and DOI.
This is the motivation for the research work presented in this paper.

While there are various issues of specific importance to either linacs or storage rings, here, we choose SSMB as an example to demonstrate the manifestations and significance of these effects. Although some of them have been studied or alluded to by other authors before in different contexts, here, we reinvestigate the relevance of these effects with an emphasis on microbunching dynamics, and some new and important results are presented. Although this analysis is oriented toward SSMB, some of the analyzed effects are also crucial in cases such as $\mathrm{CHG}$, bunch slicing, bunch compression, and FEL beam transport lines, which require precise longitudinal phase-space manipulations. These effects may also be relevant for the BESSY VSR project [13-15], the aim of which is to realize variable bunch lengths in storage rings by using different rf systems. These investigations are also expected to be useful for understanding and controlling the dynamics in extreme or advanced modes, such as the quasi-isochronous operation of a storage ring, which are essential for both the present accelerator upgrades and the general development trend of diffraction-limited storage ring design.

The paper is organized in the following manner. In Sec. II, the basic concept and promising potential of SSMB as well as recent related progress are briefly reviewed as the background for this research work. Sections III and IV then treat the dynamics in the longitudinal dimension, while Secs. V and VI are devoted to the transverse-longitudinal coupling. In both cases, we start from the linear dynamics 
and then transition to the nonlinear dynamics without considering chaos. A brief summary is finally given in Sec. VII.

\section{SSMB}

\section{A. From rf buckets to microbuckets and from bunches to microbunches}

The SSMB mechanism was proposed by Ratner and Chao [10] to generate coherent radiation of high average power in electron storage rings at wavelengths ranging from the terahertz $(\mathrm{THz})$ region to the extreme ultraviolet (EUV). The idea of SSMB is that by means of precise phase-space manipulation of the electron beam, microbunching is formed and remains in a steady state each time it passes through the radiator in a storage ring. In its most natural form, SSMB involves the replacement of the conventional radio frequency (rf) cavity for bunching with a laser modulator for microbunching. The wavelength of laser is typically six orders of magnitudes shorter than that of an rf. This modulator is usually an undulator and is much shorter than that in a high-gain FEL; therefore, there is no FEL energy heating. The SSMB mechanism is essentially a dimensional scaling of the longitudinal beam dynamics from the microwave region to optical wavelengths, from $\mathrm{rf}$ buckets to optical microbuckets, and from bunches to microbunches. The schematic layout and basic principle of SSMB and a comparison with a traditional storage ring are shown in Fig. 1.

SSMB harnesses the potential of the longitudinal coherence of the electron beam in a storage ring, parallel to the present efforts of the synchrotron radiation light source community in the context of diffraction-limited rings, which focus on the transverse dimension. The accelerator physics of SSMB pursues a similar precision in storage rings. Once SSMB is realized, the strong coherent radiation from the microbunched beam and the intrinsic high repetition rate of the storage ring can combine to support a facility with high-average-power, high-repetition-rate or continuous-wave, narrow-band, and short-wavelength radiation, which can provide unprecedented new possibilities for accelerator photon science.
Some applications do need high-average-power shortwavelength radiation. Kilowatt-level EUV light sources, for example, are urgently needed by the semiconductor industry for lithography. The potential of SSMB as a new light source mechanism is tremendous in both science and industry. Recently, an initial task force has been established at Tsinghua University, in collaboration with researchers from China, Germany, the USA, and elsewhere, to promote SSMB research with the goal of developing an SSMB storage ring $[12,16]$. The work presented in this paper is part of the ongoing investigation of SSMB [12,16-20].

\section{B. SSMB scenarios}

SSMB is a general concept with two key ingredients, i.e., microbunching and a steady state. Several specific SSMB scenarios $[10,11,21,22]$ have been proposed for different target radiation wavelengths since the first publication advancing the SSMB concept [10]. The most natural SSMB scheme, as previously mentioned, is to physically replace the rf cavity with a laser modulator to form steadystate microbunches, as shown in Fig. 1. It can be used as a laser amplifier to amplify the modulation laser power. If the microbunch is much shorter than the modulation laser wavelength, it can also be used for high harmonic generation.

To generate radiation at shorter wavelengths, for example, EUV wavelengths, two main schemes are currently being pursued by the SSMB collaboration $[12,16]$. The first approach is called longitudinal strong focusing SSMB [11]. In this scheme, a quasi-isochronous (small-momentumcompaction or low-alpha) lattice is first applied to realize and maintain the microbunch in the microbucket. The longitudinal strong focusing principle is then applied in the longitudinal dimension, not unlike quadrupole focusing in the transverse dimension, to produce an extremely short bunch at the radiator for coherent radiation generation. The second lattice approach is reversible seeding SSMB [22], in which a low-alpha lattice is not needed. This approach produces microbunching and high laser harmonics similar to those in FEL seeding techniques. However, to ensure that the microbunching process can be repeated turn by turn, the modulation and microbunching processes are perfectly
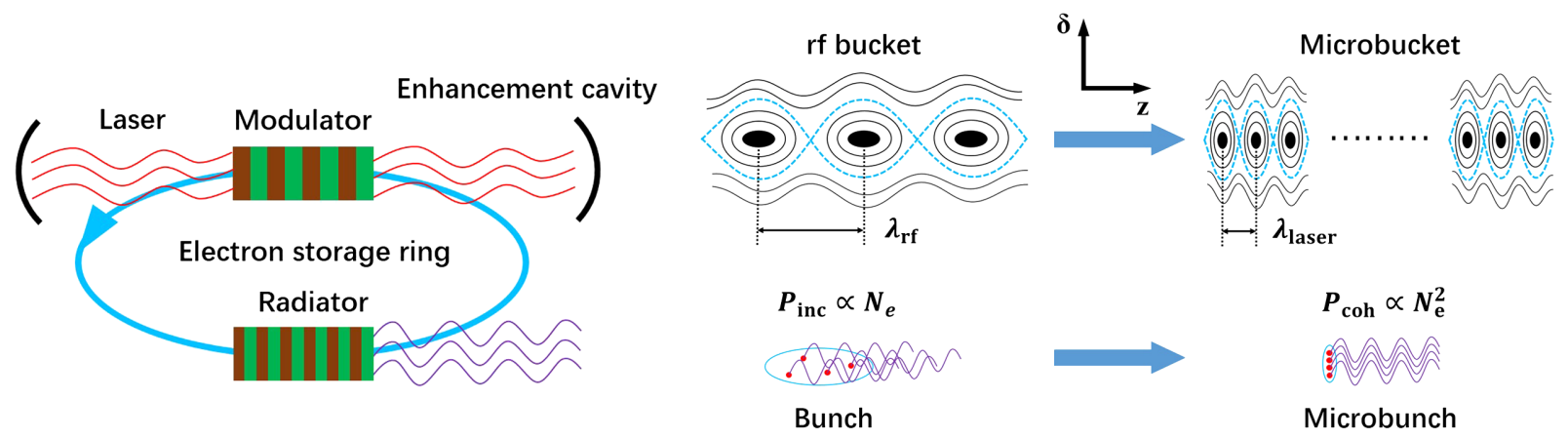

FIG. 1. Schematic layout of SSMB (left) and its operating principle in comparison to a traditional storage ring (right). 

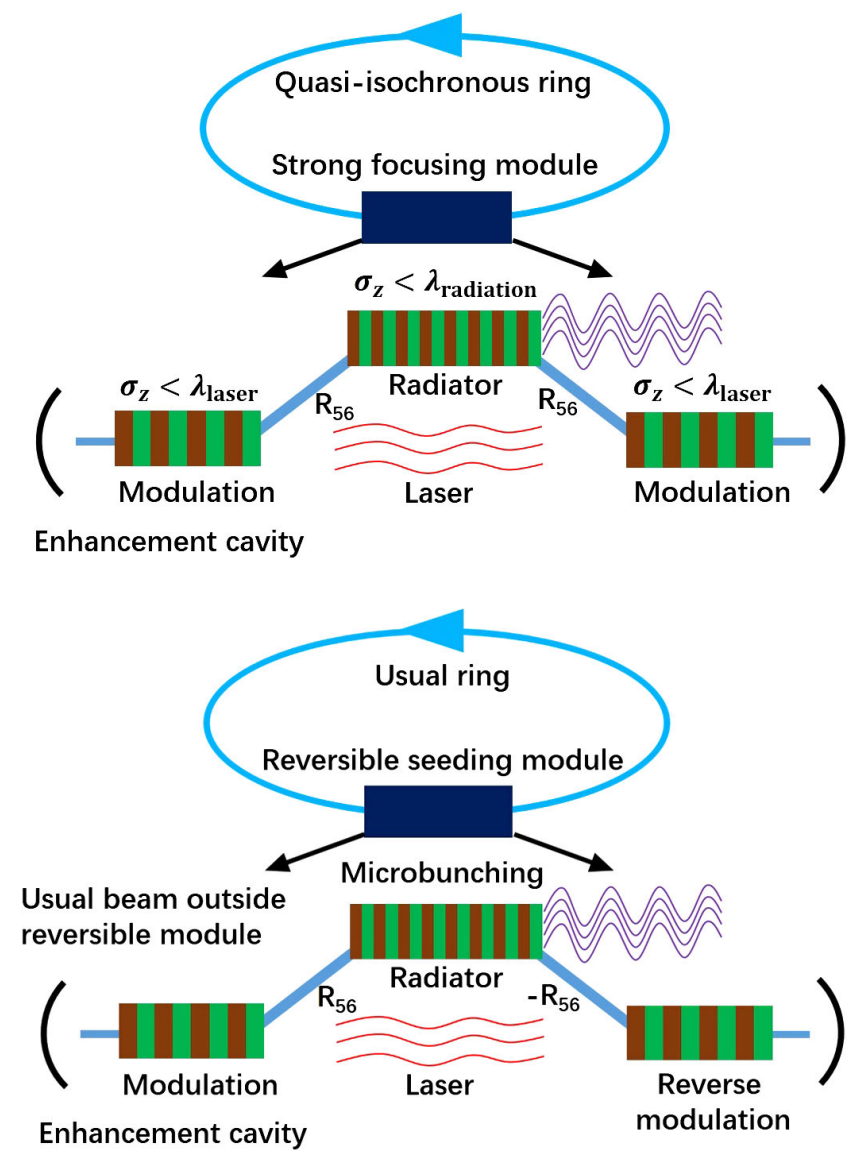

FIG. 2. Schematic layouts of longitudinal strong focusing SSMB (top) and reversible seeding SSMB (bottom).

cancelled by means of demicrobunching and reverse modulation following the radiator. Outside the reversible seeding module, the beam is identical to that in an ordinary ring. It can be a typical bunched beam or a coasting beam. This scheme relaxes the requirements on the overall lattice design outside the reversible seeding module, but the modulation cancellation requires high precision. The schematic layouts of strong focusing and reversible seeding SSMB are shown in Fig. 2.

Both approaches are being actively studied by the SSMB collaboration [18-20]. Novel 6D phase-space manipulation schemes and many interesting single-particle and collective effects can be envisioned and need to be investigated. A more in-depth study of the tricks that can be applied in lattice design and the issues related to the beam dynamics in these two SSMB scenarios will be reported in separate papers. The idea of combining the two approaches into a hybrid lattice scheme to benefit from the advantages of both is also under investigation.

\section{LONGITUDINAL QUANTUM RADIATION EXCITATION}

Some SSMB approaches, such as strong focusing $\mathrm{SSMB}$, require a low-alpha lattice. The reason for this is the well-known $\sqrt{\eta}$ scaling law for the "zero current" bunch length given by Sands [23], where $\eta$ is the global phase slippage factor of the ring, which, in the case of a high beam energy, is roughly the same as the momentum compaction factor, or the alpha. According to this scaling, it seems that all we need to generate a short bunch in a storage ring is to implement a low-alpha lattice. However, our investigations have shown that this scaling breaks down in the ultralow-alpha case, even if only the singleparticle dynamics are considered. One of the reasons is the longitudinal quantum radiation excitation, which was first theoretically analyzed by Shoji et al. [24] Here, we start by explaining the physical picture of the longitudinal excitation and then present a simplified physical derivation of new bunch length and energy spread formulas that account for the main physics of this effect. Numerical simulations conducted using the lattice of a real machine are reported thereafter to validate the analysis. Finally, methods of mitigating this effect are discussed, and the scaling laws of this effect with respect to the particle energy and the bending magnet characteristics are introduced.

\section{A. Physical picture}

The key to understanding this effect is to shift from the global perspective to a local one. In Sands' analysis, the quantum excitation in the longitudinal dimension is treated as a lumped element, considering only the excitation of the particle energy in each turn. However, photon emission is actually a random process that can occur anywhere there is a bending magnet. There will also be an excitation in the longitudinal coordinate in each turn; indeed, this is the reason that the name longitudinal excitation was coined, because the energy losses at different positions will lead to one-turn path-length differences due to the variation in the partial phase slippage. The partial phase slippage from the $j$ th photon emission point $s_{j}$ to the observation point $s_{o}$ is defined as

$$
\tilde{\eta}\left(s_{j}, s_{o}\right)=\frac{1}{C_{0}} \int_{s_{j}}^{s_{o}}\left(\frac{D_{x}(s)}{\rho(s)}-\frac{1}{\gamma_{0}^{2}}\right) d s
$$

where $C_{0}$ is the ring circumference, $D_{x}$ is the horizontal dispersion, $\rho$ is the bending radius and $\gamma_{0}$ is the Lorentz factor of the reference particle. Due to the stochastic nature of the photon emission process, this diffusion in bunch length is unavoidable even if the global phase slippage is zero because we cannot make all of the partial phase slippages zero simultaneously. Clearly, then, Sands' analysis will break down at some point. The physical pictures of the partial phase slippage and the quantum excitation in both the energy and the longitudinal coordinate in each turn are shown in Fig. 3. 


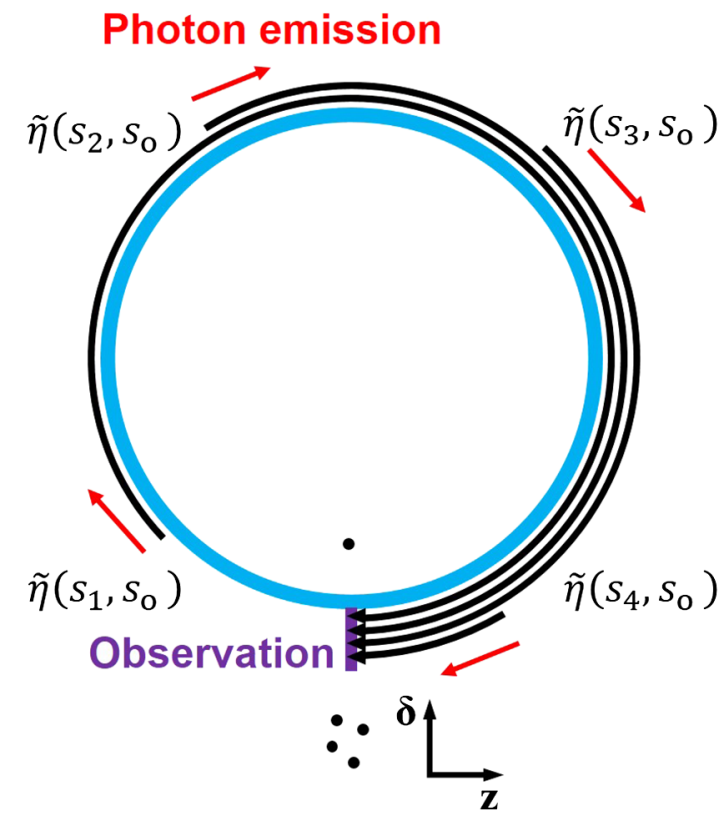

FIG. 3. Physical pictures of partial phase slippage and quantum excitation. Particles undergo diffusion in both the particle energy and the longitudinal coordinate in each turn, giving rise to longitudinal emittance, as a result of quantum excitation.

\section{B. A simplified physical derivation}

For an electron storage ring, the main consequence of this effect is that there exists a steady-state bunch length limit given by

$$
\sigma_{z \mathrm{~L}}=C_{0} \sqrt{I_{\tilde{\eta}}} \sigma_{\delta \mathrm{S}},
$$

with $I_{\tilde{\eta}}=\left\langle\left[\tilde{\eta}\left(s_{j}, s_{o}\right)-\left\langle\tilde{\eta}\left(s_{i}, s_{o}\right)\right\rangle\right]^{2}\right\rangle$ being the variance of the partial phase slippage and

$$
\sigma_{\delta \mathrm{S}}=\sqrt{\frac{\left\langle N \frac{u^{2}}{E_{0}^{2}}\right\rangle}{4 k_{s} T_{0}}}
$$

being the classical Sands energy spread [23], where $N$ is the average number of photons emitted in one turn, $u$ is the emitted photon energy, $E_{0}$ is the electron energy, $k_{s}$ is the longitudinal radiation damping coefficient and $T_{0}$ is the revolution period. The weight of each position in the statistics is determined by the probability of radiation at that position. For an isomagnetic ring, this limit can be calculated once the dispersion function pattern and the operating energy of the ring are given. This limit shows little dependence on the global phase slippage factor $\eta$ or the rf voltage gradient $V_{\mathrm{rf}}^{\prime}=d V_{\mathrm{rf}} / d t$.

The result in Eq. (2) can be obtained in a manner similar to that in which the energy spread is derived in Sands' theory [23]. The diffusion of the longitudinal coordinate in one turn due to the longitudinal excitation illustrated in Fig. 3 is

$$
d_{z \mathrm{~L}}=C_{0} \sqrt{\left\langle N \frac{u^{2}}{E_{0}^{2}}\right\rangle I_{\tilde{\eta}}}
$$

The steady-state bunch length limit is the diffusion in one turn multiplied by the square root of the damping time in a unit of number of turns and divided by 2 ,

$$
\sigma_{z \mathrm{~L}}=\frac{1}{2} d_{z \mathrm{~L}} \sqrt{\frac{1}{k_{s} T_{0}}}=C_{0} \sqrt{I_{\tilde{\eta}}} \sigma_{\delta \mathrm{S}},
$$

which is the result shown in Eq. (2).

Considering the longitudinal excitation and ignoring the transverse-longitudinal coupling and higher-order terms, Sands' formula for the bunch length should be modified to

$$
\sigma_{z}=\sqrt{\sigma_{z \mathrm{~S}}^{2}+\sigma_{z \mathrm{~L}}^{2}}=C_{0} \sqrt{\frac{E_{0}|\eta|}{e V_{\mathrm{rf}}^{\prime} T_{0}}+I_{\tilde{\eta}}} \sigma_{\delta \mathrm{S}},
$$

where $e$ is the elementary charge. Because the rf cavity will convert the diffusion of the arrival time into differences in particle energy, this effect also contributes to the energy spread,

$$
\sigma_{\delta}=\sqrt{\sigma_{\delta \mathrm{S}}^{2}+\sigma_{\delta \mathrm{L}}^{2}}
$$

Due to the rf focusing, which is the same for different diffusion sources, we have

$$
\frac{\sigma_{z \mathrm{~S}}}{\sigma_{\delta \mathrm{S}}}=\frac{\sigma_{z \mathrm{~L}}}{\sigma_{\delta \mathrm{L}}}
$$

and the energy spread in Eq. (7) can thus be expressed as

$$
\sigma_{\delta}=\sigma_{\delta \mathrm{S}} \sqrt{1+\left(\frac{\sigma_{z \mathrm{~L}}}{\sigma_{z \mathrm{~S}}}\right)^{2}},
$$

and, more specifically, as

$$
\sigma_{\delta}=\sigma_{\delta \mathrm{S}} \sqrt{1+\frac{e V_{\mathrm{rf}}^{\prime} T_{0} I_{\tilde{\eta}}}{E_{0}|\eta|}}
$$

Equations (6) and (10) are the new bunch length and energy spread formulas, in which the main physics of the longitudinal excitation is taken into account. In this paper, we call them the Shoji formulas, although they are slightly different from those presented in the original publication of Shoji et al. [24] They can also be more rigorously derived using the Chao-Lee method of solving the Fokker-Planck equation [25], which will be presented in another paper along with an analysis of various additional noise sources in storage rings. 
In fact, what this effect emphasizes is that we should consider the influence of the quantum excitation on the longitudinal emittance rather than that on the particle energy alone. The analysis of Shoji et al. [24] and the simplified derivation in this paper are both based on certain assumptions regarding the lattice; for example, it is assumed that the ring is planar and consists of isomagnets. Some approximations have also been made to obtain the final concise results of Eqs. (6) and (10) to allow this effect to be conveniently evaluated in terms of a single parameter $I_{\tilde{\eta}}$. For a more rigorous analysis of this effect in a general linearly coupled lattice, one can use the method of Nash [26],

$$
\Delta\left\langle\epsilon_{k}\right\rangle=\oint d s \operatorname{Tr}\left[\mathbf{G}_{k} \frac{d \mathbf{\Sigma}}{d s}\right],
$$

to calculate the change in emittance in one turn based on the transfer map and the diffusion process. Chao's SLIM formalism [27] and the beam envelope matrix method of Ohmi et al. [28] are also the accurate approaches for treating this effect. However, in most cases, the assumptions adopted in this analysis are valid, and the variance of partial phase slippage $I_{\tilde{\eta}}$ is accurate enough to be used as a criterion for quantifying this effect. Furthermore, it is more straightforward to use the familiar dispersion function language when doing the lattice design to control this effect, and Eqs. (6) and (10) are often sufficient.

\section{Simulation}

To provide a more concrete illustration of the significance and importance of this effect in realizing short bunches in electron storage rings, here, we consider the low-alpha user optics of a real machine, the Metrology Light Source (MLS) storage ring [29,30] of the Physikalisch-Technische Bundesanstalt (PTB) in Berlin, as an example for illustration. The main parameters of the MLS used in the simulation are shown in Table I.

From the numbers in Table I, the diffusion of the longitudinal coordinate in one turn and the steady-state bunch length limit due to longitudinal excitation can be calculated to be $d_{z \mathrm{~L}}=260 \mathrm{~nm}$ and $\sigma_{z \mathrm{~L}}=34 \mu \mathrm{m}$, respectively, at an operating energy of $630 \mathrm{MeV}$. The energy

TABLE I. Parameters of the MLS low-alpha user lattice.

\begin{tabular}{ll}
\hline \hline Parameter & \multicolumn{1}{c}{ Value } \\
\hline Ring circumference $C_{0}$ & $48 \mathrm{~m}$ \\
Maximum beam energy $E_{0}$ & $630 \mathrm{MeV}$ \\
rf frequency $f_{\mathrm{rf}}$ & $500 \mathrm{MHz}$ \\
Horizontal emittance $\epsilon_{x 0}$ & $200 \mathrm{~nm} @ 630 \mathrm{MeV}$ \\
Longitudinal damping time $\tau_{\delta}$ & $11 \mathrm{~ms} @ 630 \mathrm{MeV}$ \\
Natural energy spread $\sigma_{\delta \mathrm{S}}$ & $4.4 \times 10^{-4} @ 630 \mathrm{MeV}$ \\
Partial phase slippage variance $I_{\tilde{\eta}}$ & $2.6 \times 10^{-6}$ \\
\hline \hline
\end{tabular}
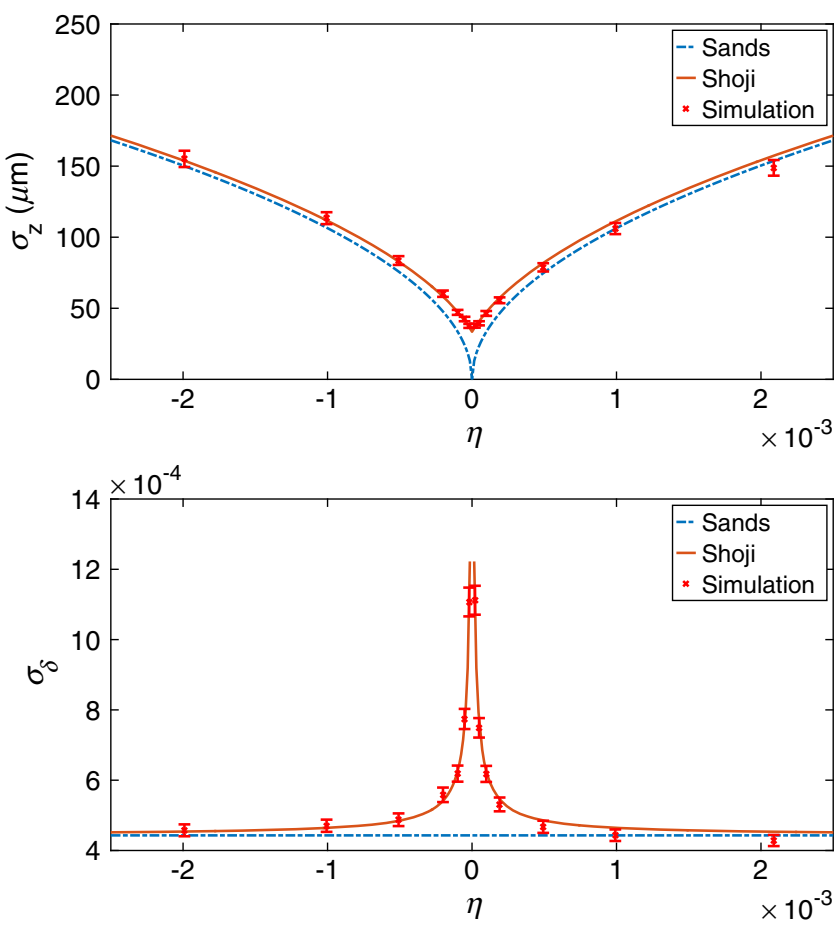

FIG. 4. Bunch length saturation and energy widening as the global phase slippage factor $\eta$ approaches zero as a result of longitudinal quantum radiation excitation. The simulation was conducted using the parameters of the MLS low-alpha user lattice at a beam energy of $630 \mathrm{MeV}$ and with the application of an extremely high rf voltage of $50 \mathrm{MV}$. A single particle was tracked for $2 \times 10^{7}$ turns, corresponding to approximately 291 longitudinal damping times, using the simulation code ELEGANT [31] to obtain the statistical bunch length and energy spread in each simulation.

spread grows significantly as the bunch length approaches the limit $\sigma_{z \mathrm{~L}}$; in the simulation, this is achieved by lowering the magnitude of the global phase slippage factor while applying an extremely high rf voltage of $50 \mathrm{MV}$. As seen in Fig. 4, the Shoji formulas agree well with the particle tracking results, while the Sands formulas fail in the case of extremely low phase slippage. Similar simulation results are presented in the dissertation of Ries [30].

\section{Methods of mitigating longitudinal excitation}

It is clear that this effect needs to be carefully treated for the realization and long-term maintenance of ultrashort bunches in either a multipass device or a single-pass transport line with bending magnets and large dispersion. It can be seen from Eqs. (4) and (5) that the significance of this effect is determined by the operating energy and the variance of the partial phase slippage, or, more loosely, the partial alpha. A lower operating energy is preferred for suppressing this quantum diffusion. Note that the energy scaling laws for this effect are different in the one-turn (single-pass) and steady-state cases; for the one-turn case, 
$d_{z \mathrm{~L}} \propto \gamma^{2.5}$, while for the steady-state case, $\sigma_{z \mathrm{~L}} \propto \gamma$, because the damping time also depends on $\gamma$.

In addition to ensuring a small global alpha, the variation in the partial alpha should also be well controlled by means of dedicated lattice design for certain SSMB scenarios. At the MLS, the small global alpha of the user optics is achieved by means of an overall integration cancellation between the large positive and large negative horizontal dispersions at different dipoles [30]. Therefore, the partial alpha varies sharply within the dipoles, leading to a large partial-alpha variance $I_{\tilde{\alpha}}$ and significant longitudinal excitation, as seen in the simulation. To obtain small global and partial alphas simultaneously, such cancellation should be achieved as locally as possible, and the magnitudes of the dispersion at the dipoles should also be minimized, thus making the partial alpha vary as gently as possible. In other words, each partial component of the ring should be made as isochronous as possible. This requirement on the dispersion is similar to that in low-emittance ring design. The partial-alpha variance $I_{\tilde{\alpha}}$ with respect to the longitudinal dimension here plays a role similar to that of the chromatic invariant $\mathcal{H}=\gamma D^{2}+$ $2 \alpha D D^{\prime}+\beta D^{\prime 2}$ in the transverse dimension. Following steps similar to those of the theoretical minimum emittance [32] analysis in the transverse dimension, for a ring consisting of identical isochronous cells, the steady-state bunch length limit [33]

$$
\sigma_{z \mathrm{~L}}=C_{0} \sqrt{I_{\tilde{\alpha}}} \sigma_{\delta \mathrm{S}} \propto \gamma \sqrt{\rho} \theta^{3}
$$

and the theoretical minimum longitudinal emittance

$$
\epsilon_{z \mathrm{M}}=2 C_{0} \sqrt{I_{\tilde{\alpha}}} \sigma_{\delta \mathrm{S}}^{2} \propto \gamma^{2} \theta^{3}
$$

can be derived, where $\rho$ and $\theta$ are the bending radius and angle, respectively, of each bending dipole magnet. According to these scaling laws, a ring consisting of a larger number of isochronous cells, each with a smaller bending angle, is better at mitigating this effect than a ring consisting of fewer cells with larger bending. Generally, it is easier to control this effect for a larger ring. Increasing the longitudinal damping partition number is also a method of suppressing this effect. Transverse and longitudinal gradient magnets can be envisioned for this purpose. However, this method may not be as effective as tuning the partial-alpha variance since the typical longitudinal damping partition number is approximately 2 , and the upper limit is 4 . Further details on the lattice design for mitigating this effect can be found in $[18,19]$.

\section{NONLINEAR MOMENTUM COMPACTION FOR HIGH HARMONIC BUNCHING}

\section{A. Physical picture}

In the low-alpha approaches for SSMB, after resolving the issue of longitudinal excitation by means of dedicated lattice design, we can then apply the low-alpha method to realize short bunches. However, the momentum compaction is actually a function of the particle energy

$$
\alpha(\delta)=\alpha_{0}+\alpha_{1} \delta+\alpha_{2} \delta^{2}+\cdots,
$$

where $\delta=\left(E-E_{0}\right) / E_{0}$ is the relative energy deviation with respect to the ideal particle. When alpha is sufficiently small, the higher-order terms in Eq. (14) may become dominant, and the beam dynamics can be significantly different from those in a linear-alpha state. Proper application of dedicated sextupoles and octupoles may be needed to control these higher-order terms.

The single-particle and collective beam dynamics of the low-alpha rings have been studied by many authors since the proposal of [34]. Here, we wish to emphasize a further point that has not previously been well investigated and might be important in some cases, for example, in the SSMB proof-of-principle experiment being conducted at the MLS $[12,17]$.

For seeding techniques such as $\mathrm{CHG}$ and high-gain harmonic generation (HGHG) $[35,36]$, it seems that to date, linear momentum compaction has been applied for microbunching formation. Here, we wish to point out that one can actually take advantage of the nonlinearity of the momentum compaction for high harmonic generation. Intuitively, this is because a sinusoidal energy modulation followed by a nonlinear dispersion can lead to a distorted current distribution, which, in some cases, can lead to high bunching at a specific harmonic number. Figure 5 shows a simulated example of the use of $\alpha(\delta)=\alpha_{1} \delta$ for microbunching. It can be seen that there is significant bunching in the second and fourth harmonics, while no bunching is
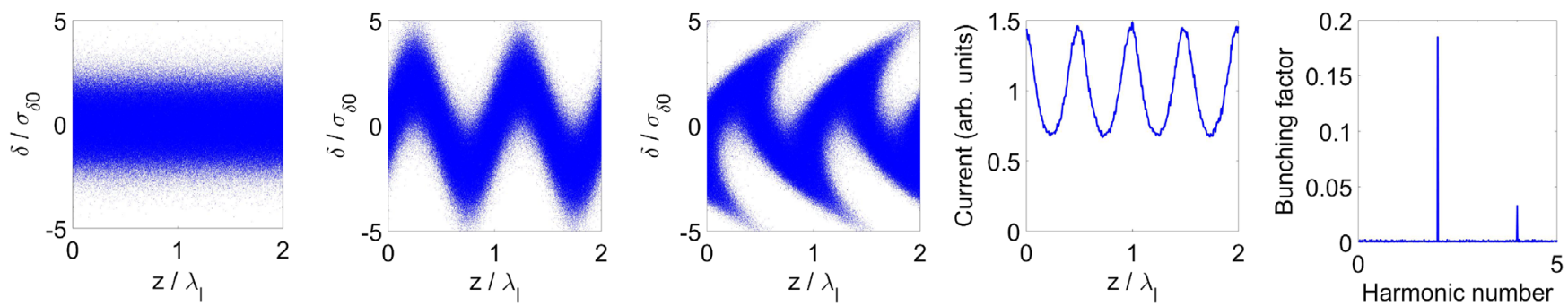

FIG. 5. The longitudinal phase-space evolution, final current distribution and bunching factor when $\alpha(\delta)=\alpha_{1} \delta$ is used for microbunching in CHG or HGHG, as modeled by Eq. (15). 
produced in the fundamental and third harmonics. The reason can be found from the following derivation of the bunching factor.

\section{B. Derivation of bunching factor}

The microbunching process in the case of a single energy modulation plus a dispersion section, as in the CHG and HGHG, can be modeled as [35]

$$
\begin{aligned}
& \delta^{\prime}=\delta+A \sigma_{\delta 0} \sin \left(k_{l} z\right) \\
& z^{\prime}=z-\alpha\left(\delta^{\prime}\right) C_{0} \delta^{\prime}
\end{aligned}
$$

where $k_{l}=2 \pi / \lambda_{l}$ is the wave number of the modulation laser, $\sigma_{\delta 0}$ is the initial energy spread and $A$ is the energy modulation depth. The bunching factor at the wave number of $k$ is defined by

$$
b(k)=\frac{1}{N_{0}}\left|\left\langle e^{-i k z^{\prime}} N\left(z^{\prime}\right)\right\rangle\right|,
$$

where $N\left(z^{\prime}\right)$ is the longitudinal particle density, $N_{0}$ is the total number of particles and the angular brackets denote averaging over the longitudinal coordinate,

$$
\langle\cdots\rangle=\lim _{L \rightarrow \infty} \frac{1}{2 L} \int_{-L}^{L} \cdots d z^{\prime}
$$

According to Liouville's theorem, we have $d z d \delta=d z^{\prime} d \delta^{\prime}$. Therefore, the bunching factor can be calculated in accordance with the initial distribution of the particle energy $f_{0}(\delta)$ as $[35,37,38]$

$$
b(k)=\frac{1}{N_{0}}\left|\int_{-\infty}^{\infty} d \delta^{\prime}\left\langle e^{-i k z^{\prime}} f_{f}\left(z^{\prime}, \delta^{\prime}\right)\right\rangle\right|=\frac{1}{N_{0}}\left|\int_{-\infty}^{\infty} d \delta f_{0}(\delta)\left\langle e^{-i k z^{\prime}(z, \delta)}\right\rangle\right|
$$

For nonlinear momentum compaction, we first consider the simple case of $\alpha(\delta) \equiv \alpha_{0}+\alpha_{1} \delta$, then

$$
z^{\prime}=z-\left[\alpha_{0}+\alpha_{1}\left(\delta+A \sigma_{\delta 0} \sin \left(k_{l} z\right)\right)\right] C_{0}\left(\delta+A \sigma_{\delta 0} \sin \left(k_{l} z\right)\right)
$$

and

$$
b(k)=\frac{1}{N_{0}}\left|\int_{-\infty}^{\infty} d \delta e^{i k\left(\alpha_{0} C_{0} \delta+\alpha_{1} C_{0} \delta^{2}+\frac{\alpha_{1} C_{0} A^{2} \sigma_{\delta 0}^{2}}{2}\right)} f_{0}(\delta)\left\langle e^{i k\left[-z+\left(\alpha_{0} C_{0} A \sigma_{\delta 0}+2 \alpha_{1} C_{0} \delta A \sigma_{\delta 0}\right) \sin \left(k_{l} z\right)-\frac{\alpha_{1} C_{0} A^{2} \sigma_{\delta 0}^{2}}{2} \cos \left(2 k_{l} z\right)\right]}\right\rangle\right| .
$$

Adopting the notation $U=k\left(\alpha_{0} C_{0} A \sigma_{\delta 0}+2 \alpha_{1} C_{0} \delta A \sigma_{\delta 0}\right), W=-k \alpha_{1} C_{0} A^{2} \sigma_{\delta 0}^{2} / 2$, and using the Fourier-Bessel expansion

$$
e^{i x \sin \left(k_{l} z\right)}=\sum_{n=-\infty}^{\infty} e^{i n k_{l} z} J_{n}[x]
$$

we have

$$
e^{-i k z+i U \sin \left(k_{l} z\right)+i W \cos \left(2 k_{l} z\right)}=\sum_{p=-\infty}^{\infty} \sum_{q=-\infty}^{\infty} J_{p}[U] J_{q}[W] \exp \left(-i\left[\left(k-(p-2 q) k_{l}\right) z-q \frac{\pi}{2}\right]\right) .
$$

Because the angular brackets represent averaging over the longitudinal coordinate and under the assumption that the bunch length is much longer than the modulation laser wavelength, $\left\langle\exp \left(-i\left[\left(k-(p-2 q) k_{l}\right) z-q \frac{\pi}{2}\right]\right)\right\rangle$ will not vanish only if $k=(p-2 q) k_{l}$ [38]. Therefore, the bunching factor at the $n$th harmonic of the modulation laser can be calculated to be

$$
b_{n}=\frac{1}{N_{0}}\left|\int_{-\infty}^{\infty} d \delta e^{i n k_{l}\left(\alpha_{0} C_{0} \delta+\alpha_{1} C_{0} \delta^{2}+\frac{\alpha_{1} C_{0} A^{2} \sigma_{\delta 0}^{2}}{2}\right)} f_{0}(\delta) \sum_{m=-\infty}^{\infty} J_{n+2 m}[U] J_{m}[W]\right|
$$

Because $U$ is also dependent on $\delta$, it is difficult to obtain a general concise analytical formula for the above expression. In this paper, we consider only the simple case of an initial Gaussian energy distribution $f_{0}(\delta)=$ $\frac{N_{0}}{\sqrt{2 \pi} \sigma_{\delta 0}} \exp \left(-\frac{\delta^{2}}{2 \sigma_{\delta 0}^{2}}\right)$.

If $\quad \alpha_{1}=0$, then $U=n k_{l} \alpha_{0} C_{0} A \sigma_{\delta 0}, \quad W=0, \quad$ and $\sum_{m=-\infty}^{\infty} J_{n+2 m}[U] J_{m}[W]=J_{n}\left[n k_{l} \alpha_{0} C_{0} A \sigma_{\delta 0}\right]$, meaning that we have

$$
b_{n}=\left|J_{n}\left[n k_{l} \alpha_{0} C_{0} A \sigma_{\delta 0}\right] \exp \left(-\frac{\left(n k_{l} \alpha_{0} C_{0} \sigma_{\delta 0}\right)^{2}}{2}\right)\right|,
$$

which is a familiar result for HGHG if we adopt the notation $R_{56}=-\alpha_{0} C_{0}$ [35]. If $\alpha_{0}=0$, then $U=2 n k_{l} \alpha_{1} C_{0} \delta A \sigma_{\delta 0}, \quad W=-n k_{l} \alpha_{1} C_{0} A^{2} \sigma_{\delta 0}^{2} / 2$, meaning that we have 


$$
b_{n}=\frac{1}{\sqrt{2 \pi} \sigma_{\delta 0}}\left|\int_{-\infty}^{\infty} d \delta \exp \left[i n k_{l}\left(\alpha_{1} C_{0} \delta^{2}+\frac{\alpha_{1} C_{0} A^{2} \sigma_{\delta 0}^{2}}{2}\right)\right] \exp \left(-\frac{\delta^{2}}{2 \sigma_{\delta 0}^{2}}\right) \sum_{m=-\infty}^{\infty} J_{n+2 m}[U] J_{m}[W]\right| .
$$

The two exponential terms in the integral are even functions of $\delta$, while $J_{n+2 m}[U] J_{m}[W]$ is an odd function of $\delta$ when $n$ is odd; thus, $b_{n}$ is nonzero only for even $n$. This is why bunching occurs only in the second and fourth harmonics but not in the fundamental and third harmonics when we use $\alpha(\delta)=\alpha_{1} \delta$ for microbunching, as shown in Fig. 5.

\section{Discussion}

Following the derivations and according to the relation

$$
\cos ^{n}(x)=\frac{1}{2^{n-1}} \sum_{m=(n+1) / 2}^{n}\left(\begin{array}{l}
n \\
m
\end{array}\right) \cos (2 m-n) x,
$$

it can be seen that the energy modulation at the fundamental frequency can be cast as $[i \times(n-2 p)+j \times(n-2 q)]$ thharmonic bunching through the term $\alpha_{n-1} \delta^{n}$ in the alpha function $\alpha(\delta)$. The optimal bunching condition for a specific harmonic requires the matching of the alpha function with the modulation depth. However, the analytical formula for the bunching factor will become increasingly involved as more higher-order terms of the momentum compaction are considered. Thus, it would be better to rely on numerical integration and simulation to calculate and optimize the bunching factor directly for a specific application case. For storage rings, another relevant point is that the distribution of the particle energy in the nonlinear-alpha state may also have an impact on the high harmonic generation, and this phenomenon is also easier to study by means of numerical simulation.

The approach of applying a nonlinear alpha for high harmonic bunching can be considered to share some similarities with echo-enabled harmonic generation (EEHG) [37,38], in which the sinusoidal energy modulation and dispersion in the first stage can be viewed as the source of the distorted current distribution in the second stage of modulation and dispersion for microbunching. We have also noticed the work of Stupakov and Zolotorev on optimizing the nonlinearity of the dispersion to increase the bunching factor for EEHG [39]. Based on similar considerations, tricks can also be applied on the energymodulation waveform side using different harmonics of the modulation laser, for example, forming a sawtooth waveform to boost bunching, as proposed in [39-41].

\section{LINEAR TRANSVERSE-LONGITUDINAL COUPLING}

In the above two sections, we have restricted the analysis to the longitudinal dimension alone. In this section, we treat the transverse-longitudinal coupling dynamics in the linear case. Nonlinear coupling will be studied in the next section.

\section{A. Physical picture}

In a linear transport line without bending magnets, the transverse and longitudinal motions are decoupled in a first-order approximation. However, the situation changes when there are bending magnets. Particles with different horizontal (vertical) positions and angles will pass through the horizontal (vertical) bending magnets along different paths, resulting in differences in the longitudinal coordinate. The transverse motion can thus be coupled to the longitudinal dimension. When traversing the bending magnets, particles with different energies will also pass along different paths and exit with different horizontal (vertical) positions and angles. The longitudinal motion can thus also be coupled to the transverse dimension through the dispersion $D$ and dispersion angle $D^{\prime}$ induced by the bending magnets. The physical pictures of the linear transverse-longitudinal coupling introduced by the bending
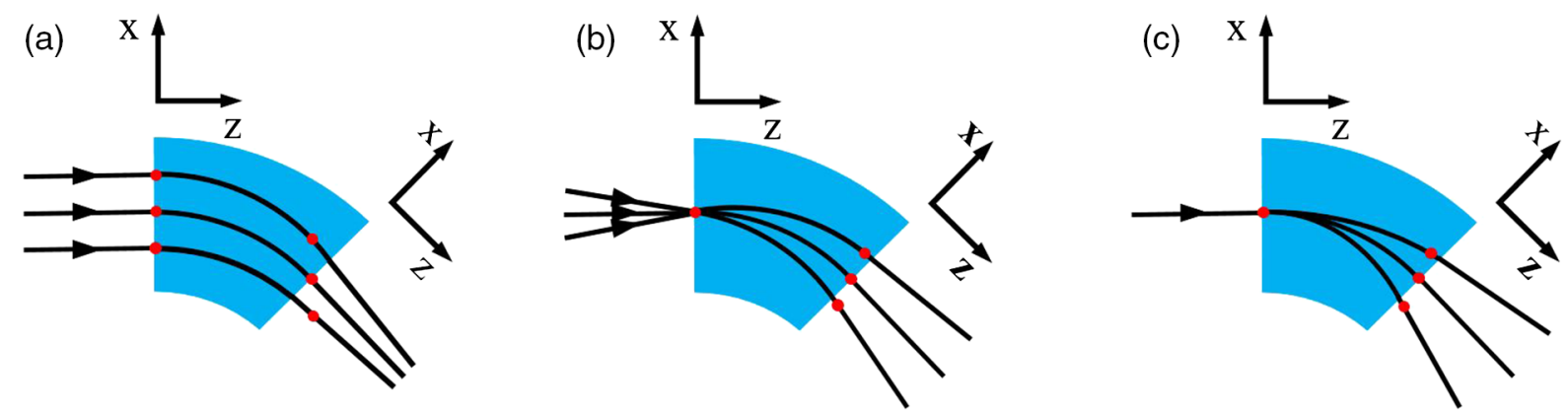

FIG. 6. Linear transverse-longitudinal coupling induced by a bending magnet. Particles with different horizontal positions (a) and angles (b) pass the horizontal bending magnet along different paths, resulting in longitudinal coordinate differences. Particles with different energies (c) also pass the horizontal bending magnet along different paths, resulting in horizontal position and angle differences. 
magnets are shown in Fig. 6. Although this is a wellunderstood effect [42-46], here, we present a concise analysis of this effect with an emphasis on its vital role in microbunching formation and transportation for both the transient and steady-state cases.

\section{B. Derivation}

We start with a planar uncoupled lattice, temporarily ignoring the vertical dimension. The state vector is $\mathbf{X}=\left(x, x^{\prime}, z, \delta\right)^{T}$, with ${ }^{T}$ representing the transpose. The subscripts 5,6 are used for $z, \delta$ for consistency with the literature. Hereafter, the subscript of ${ }_{x}$ is omitted unless necessary.

The betatron coordinate, defined by $\mathbf{X}_{\beta}=\mathbf{B X}$, is first used to parametrize the transport matrix in diagonal form. The dispersion matrix $\mathbf{B}$ is given by

$$
\mathbf{B}=\left(\begin{array}{cccc}
1 & 0 & 0 & -D \\
0 & 1 & 0 & -D^{\prime} \\
D^{\prime} & -D & 1 & 0 \\
0 & 0 & 0 & 1
\end{array}\right)
$$

The transport matrix for $\mathbf{X}_{\beta}$ of a lattice from $s_{1}$ to $s_{2}$ without an rf kick in between is then

$$
\mathbf{M}_{\beta}\left(s_{1}, s_{2}\right)=\left(\begin{array}{cc}
\mathbf{M}_{x_{\beta}}\left(s_{1}, s_{2}\right) & \mathbf{0} \\
\mathbf{0} & \mathbf{M}_{z_{\beta}}\left(s_{1}, s_{2}\right)
\end{array}\right),
$$

with

$$
\mathbf{X}_{\beta}\left(s_{2}\right)=\mathbf{M}_{\beta}\left(s_{1}, s_{2}\right) \mathbf{X}_{\beta}\left(s_{1}\right) .
$$

Following Courant and Snyder [47], we write $\mathbf{M}_{x_{\beta}}\left(s_{1}, s_{2}\right)$ as

$$
\mathbf{M}_{x_{\beta}}\left(s_{1}, s_{2}\right)=\mathbf{A}^{-1}\left(s_{2}\right) \mathbf{T}\left(s_{1}, s_{2}\right) \mathbf{A}\left(s_{1}\right),
$$

with

$$
\begin{aligned}
\mathbf{M}\left(s_{1}, s_{2}\right) & =\left(\begin{array}{cccc}
R_{11} & R_{12} & 0 & D_{2}-R_{11} D_{1}-R_{12} D_{1}^{\prime} \\
R_{21} & R_{22} & 0 & D_{2}^{\prime}-R_{21} D_{1}-R_{22} D_{1}^{\prime} \\
R_{51} & R_{52} & 1 & F-R_{51} D_{1}-R_{52} D_{1}^{\prime} \\
0 & 0 & 0 & 1
\end{array}\right) \quad R_{11}=\sqrt{\frac{\beta_{2}}{\beta_{1}}\left[\cos \psi_{12}+\alpha_{1} \sin \psi_{12}\right]} \\
R_{12} & =\sqrt{\beta_{1} \beta_{2}} \sin \psi_{12} \quad R_{21}=-\frac{1}{\sqrt{\beta_{1} \beta_{2}}}\left[\left(1+\alpha_{1} \alpha_{2}\right) \sin \psi_{12}-\left(\alpha_{1}-\alpha_{2}\right) \cos \psi_{12}\right] \\
R_{22} & =\sqrt{\frac{\beta_{1}}{\beta_{2}}}\left[\cos \psi_{12}-\alpha_{2} \sin \psi_{12}\right] \quad R_{51}=R_{21} D_{2}-R_{11} D_{2}^{\prime}+D_{1}^{\prime} \quad R_{52}=-R_{12} D_{2}^{\prime}+R_{22} D_{2}-D_{1} .
\end{aligned}
$$

This matrix can then be used to analyze both the transient and steady-state cases. 
We first consider the influence of the betatron oscillation alone on the longitudinal coordinate. With the help of the Courant-Snyder formalism, the betatron oscillation position and angle at the starting point $s_{1}$ can be expressed as

$$
\begin{aligned}
& x_{1}=\sqrt{2 J \beta_{1}} \cos \psi_{1} \\
& x_{1}^{\prime}=-\sqrt{2 J / \beta_{1}}\left(\alpha_{1} \cos \psi_{1}+\sin \psi_{1}\right),
\end{aligned}
$$

where

$$
J=\frac{1}{2}\left(\gamma x^{2}+2 \alpha x x^{\prime}+\beta x^{\prime 2}\right)
$$

is the betatron invariant. The longitudinal coordinate displacement relative to the ideal particle due to the betatron oscillation from $s_{1}$ to $s_{2}$ is then

$$
\begin{aligned}
\Delta z & =R_{51} x_{1}+R_{52} x_{1}^{\prime} \\
& =\sqrt{2 J \mathcal{H}_{1}} \sin \left(\psi_{1}-\chi_{1}\right)-\sqrt{2 J \mathcal{H}_{2}} \sin \left(\psi_{2}-\chi_{2}\right),
\end{aligned}
$$

where to obtain the final concise result, $D$ and $D^{\prime}$ have been expressed in terms of the chromatic invariant $\mathcal{H}$ and the chromatic phase $\chi$, defined as

$$
\begin{aligned}
& D=\sqrt{\mathcal{H} \beta} \cos \chi \quad D^{\prime}=-\sqrt{\mathcal{H} / \beta}(\alpha \cos \chi+\sin \chi) \\
& \mathcal{H}=\gamma D^{2}+2 \alpha D D^{\prime}+\beta D^{\prime 2} .
\end{aligned}
$$

If the particle starts with a relative energy deviation of $\delta$, then

$$
\begin{aligned}
\Delta z & =R_{51} x_{1}+R_{52} x_{1}^{\prime}+\left(F-R_{51} D_{1}-R_{52} D_{1}^{\prime}\right) \delta \\
& =\sqrt{2 J \mathcal{H}_{1}} \sin \left(\psi_{1}-\chi_{1}\right)-\sqrt{2 J \mathcal{H}_{2}} \sin \left(\psi_{2}-\chi_{2}\right)+F \delta .
\end{aligned}
$$

Note that in Eq. (42), the betatron invariant and phase should be calculated according to

$$
\begin{aligned}
x_{\beta} & =x-D \delta=\sqrt{2 J \beta} \cos \psi \\
x_{\beta}^{\prime} & =x^{\prime}-D^{\prime} \delta=-\sqrt{2 J / \beta}(\alpha \cos \psi+\sin \psi) \\
J & =\frac{1}{2}\left[\gamma x_{\beta}^{2}+2 \alpha x_{\beta} x_{\beta}^{\prime}+\beta x_{\beta}^{\prime 2}\right] .
\end{aligned}
$$

For a periodic system, if we observe the particle at the same place $n$ periods later, then

$\Delta z=\sqrt{2 J \mathcal{H}}\left[\sin (\psi-\chi)-\sin \left(\psi+2 n \pi \nu_{x}-\chi\right)\right]-n C_{0} \eta \delta$,

where $\nu_{x}$ is the horizontal betatron tune.

Equations (42) and (44) can be used to explain the dependence of the coherent synchrotron radiation (CSR) repetition rate on the betatron tune in the bunch slicing experiment of Shimada et al. [45] A similar approach can also be applied to analyze microbunching preservation in FEL beam transportation, for example, in the microbunching rotation experiment of Macarthur et al. [49] These equations are also useful for evaluating the influence of the coupling effect in the SSMB proof-of-principle experiment $[12,17]$. Note that when Eqs. (42) and (44) are applied for beam analysis, it is assumed that the initial dispersion $\left(D_{1}, D_{1}^{\prime}\right)$ of the lattice matches that of the beam. If this is not the case, then this mismatch should also be taken into account.

This linear transverse-longitudinal coupling will also influence the steady-state bunch distribution in a storage ring. Because the different particles in a bunch have different betatron amplitudes and phases as well as different momenta, according to Eq. (42), the total bunch length can be calculated to be

$$
\sigma_{z}=\sqrt{\sigma_{z \beta}^{2}+\epsilon_{x} \mathcal{H}_{x}}
$$

where $\sigma_{z \beta}$ is the bunch length contributed by the longitudinal emittance alone.

We can also obtain all of the second moments by following the Courant-Snyder parametrization one step further. The second moment matrix of the betatron coordinate $\mathbf{X}_{\beta}$ in the steady state is

$$
\boldsymbol{\Sigma}_{\beta}=\left(\begin{array}{cccc}
\epsilon_{x} \beta_{x} & -\epsilon_{x} \alpha_{x} & 0 & 0 \\
-\epsilon_{x} \alpha_{x} & \epsilon_{x} \gamma_{x} & 0 & 0 \\
0 & 0 & \epsilon_{z} \beta_{z} & -\epsilon_{z} \alpha_{z} \\
0 & 0 & -\epsilon_{z} \alpha_{z} & \epsilon_{z} \gamma_{z}
\end{array}\right)
$$

The second moment matrix of the physical coordinate $\mathbf{X}$ is then

$$
\boldsymbol{\Sigma}=\mathbf{B}^{-1} \boldsymbol{\Sigma}_{\beta}\left(\mathbf{B}^{-1}\right)^{T} .
$$

For a Gaussian beam, the beam distribution is determined solely by the second moment matrix according to

$$
\psi(\mathbf{X})=\frac{1}{(2 \pi)^{2} \sqrt{\operatorname{det} \mathbf{\Sigma}}} \exp \left(-\frac{1}{2} \mathbf{X}^{T} \boldsymbol{\Sigma}^{-1} \mathbf{X}\right) .
$$

We are especially interested in the impact of this effect on the formation and transportation of longitudinal microstructures. Usually, we want the momentum compaction term $F$ to be dominant in determining the longitudinal dynamics. It can be seen from Eqs. (42), (44), and (45) that the most straightforward way to eliminate this coupling effect is to let $\mathcal{H}_{1}=\mathcal{H}_{2}=0$, which corresponds to $R_{51}=R_{52}=0$. An achromatic lattice should thus be envisioned. For the transient or single-pass case with a nonzero initial dispersion, it is also possible to eliminate 
this coupling effect by tuning the chromatic invariant and matching the betatron and chromatic phase advances such that $\mathcal{H}_{2}=\mathcal{H}_{1}$ and $\psi_{2}-\psi_{1}=\chi_{2}-\chi_{1}$.

Although it increases the bunch length, this coupling effect may also be helpful for suppressing harmful CSRinduced effects in SSMB or other applications, as extremely short bunches emerge only at dispersion-free locations.

\section{Transverse-longitudinal coupling for harmonic generation and bunch length manipulation}

The above analysis may lead readers to conclude that transverse-longitudinal coupling always increases the bunch length and degrades the microbunching effect, as shown in Eqs. (40) and (45). This, however, is not true, as the above analysis is based on the assumption of a planar uncoupled lattice with only the passive coupling induced by the bending magnets. In fact, one can take advantage of the transverse-longitudinal coupling for harmonic generation or bunch compression when the transverse emittance is small. The reason is that there is some flexibility in the projection of the three eigenemittances of a beam into different physical dimensions, although their values cannot be changed in a linear symplectic lattice [50]. Emittance exchange (EEX) [51,52] is an example of such flexibility.

In addition to the passive coupling introduced by the bending magnets, which manifests in the dispersion and dispersion angle, an rf cavity (laser modulator) placed at a dispersive location is another source of coupling that can be used for subtle manipulation of the coupling dynamics.
More novel 6D phase-space gymnastics can be invoked to tailor specific parameters of the beam, such as the bunch length.

The first proposal for the application of such a transverse-longitudinal coupling scheme in FEL seeding is the phase-merging enhanced harmonic generation (PEHG) [53,54]. A PEHG unit can be divided into four key functional components: (i) a dispersion generation $\left(R_{16}=D_{x}\right)$ device, for example, a dogleg, to correlate $x$ with $\delta$; (ii) a laser modulator to generate an energy chirp $\left(R_{65}=h\right)$ to correlate $\delta$ with $z$; (iii) a dispersive section $\left(R_{56}\right)$ for longitudinal phase-space rotation to correlate $z$ with $\delta$ to form microbunching; and (iv) a phase-merging unit $\left(R_{51}=m\right)$ to correlate $z$ with $x$. The trick for phase merging is to achieve cancellation between the longitudinal displacement contribution from $\delta$ (through $R_{56}$ ) and that from $x$ introduced in the first step (through $D_{x} m$ ), thus causing the longitudinal phases of different particles to merge together to generate a very sharp current spike and high harmonic bunching.

The physical principle of PEHG, as explained above, can be illustrated with the help of the longitudinal phase-space evolution shown in Fig. 7. A thin-lens matrix analysis of the four functions reveals that to achieve optimal microbunching (bunch compression) and phase merging, we need

$$
1+R_{56} h=0 \quad R_{56}+D_{x} m=0 .
$$

In the bunching factor formula corresponding to Eq. (24) for HGHG, the effective energy spread of PEHG is then $\sigma_{\text {eff }}=\frac{\sigma_{x}}{D_{x}}$. PEHG is favorable for harmonic generation or

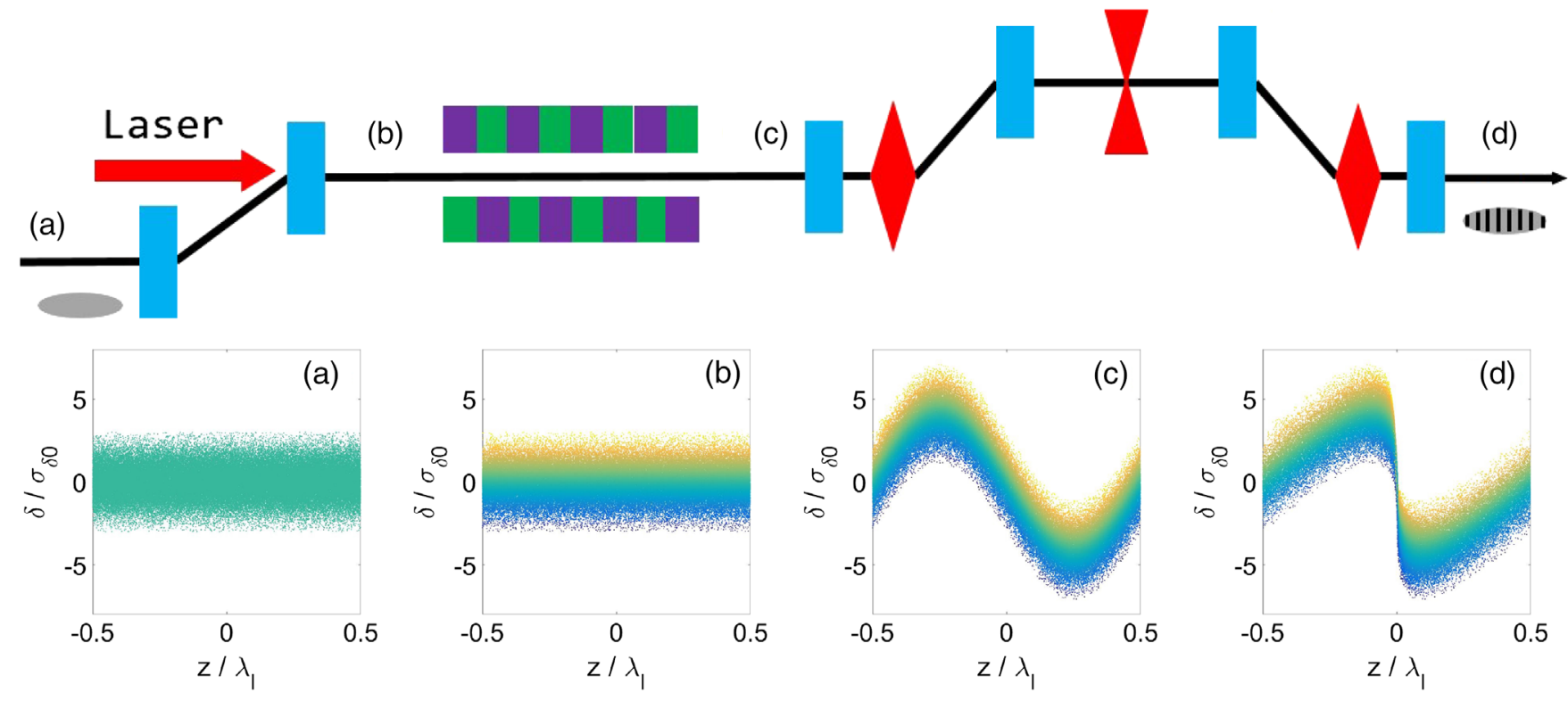

FIG. 7. Schematic layout (top) and longitudinal phase-space evolution (bottom) of the proposed PEHG variant. (a) before entering the PEHG unit, (b) after the dispersion-generation device, (c) after the energy modulation, (d) after the dispersive section with phasemerging function to form microbunching. Different colors in the phase-space plots represent different horizontal positions. Compared to the original PEHG scheme, the phase-merging function is shifted downstream to the dispersive section by introducing a transverse gradient in the bending dipoles or inserting quadrupoles in between. 
bunch compression when the transverse emittance is sufficiently small, thus that $\sigma_{\text {eff }}<\sigma_{\delta 0}$.

There are several variants of PEHG that can be advantageous in different specific cases, for example, using $D_{x^{\prime}, y, y^{\prime}}$ rather than $D_{x}$; the formulas for the effective energy spread and the final bunching factor change accordingly, but in spirit, the situation is the same as the one analyzed here. For example, in [55], a novel PEHG variant has been proposed for generating very high harmonic bunching in CHG by making use of the fact that the vertical emittance is very small and the bunch length can reach a very small value in a planar ring.

To better illustrate the mechanism, we have divided the PEHG process into four functions. Note, however, that there is some flexibility in their order. Additionally, these functions can be realized one by one, or with two or three of them simultaneously. For example, in the original publication on PEHG [53], a transverse-gradient undulator was applied along with the modulation laser to realize energy chirping and phase merging at the same time. Here, we propose a PEHG variant in which the phase-merging function is shifted downstream to the dispersive section by introducing a transverse gradient into the chicane dipoles or inserting quadrupoles into the chicane. Only a normal undulator and normal elements are needed in the proposed variant, which should make it easier to implement and tune than the original PEHG scheme. The layout and longitudinal phase-space evolution of the proposed PEHG variant are shown in Fig. 7.

If we wish to benefit from transverse-longitudinal coupling in SSMB, we need to apply PEHG on a turnby-turn basis, which is not as easy as in the single-pass case. We need to ensure that the desired distribution is indeed the eigendistribution, as a storage ring is different from a linac in that it is a multipass device. For a general coupled storage ring lattice, the Courant-Snyder formalism is no longer convenient. Chao's SLIM formalism [27] can instead be applied to perform the analysis. The 6D phasespace vector $\mathbf{X}=\left(x, x^{\prime}, y, y^{\prime}, z, \delta\right)^{T}$ is used, and the $6 \times 6$ general coupled matrices are our starting point. From SLIM, we know that

$$
\Sigma_{i j}=\left\langle X_{i} X_{j}\right\rangle=2 \sum_{k=I, I I, I I I} \epsilon_{k} \operatorname{Re}\left(E_{k i} E_{k j}^{*}\right),
$$

where $\epsilon_{k}$ are the eigenemittances and $\mathbf{E}_{k}$ are the eigenvectors of the linear one-turn map M. Then,

$$
\sigma_{i}^{2}=\Sigma_{i i}=2 \sum_{k=I, I I, I I I} \epsilon_{k}\left|E_{k i}\right|^{2} .
$$

Equation (51) means that the three eigenemittances contribute independently to $\sigma_{i}^{2}$ and that the projection weights are determined by the $i$ th components of the eigenvectors $\mathbf{E}_{k}$. It can be seen from Eq. (45) that for a planer uncoupled lattice, the weights of the longitudinal and horizontal emittances in the longitudinal bunch length are the longitudinal beta function $\beta_{z}$ and the horizontal chromatic function $\mathcal{H}_{x}$, respectively.

The power of a lattice with transverse-longitudinal coupling lies in the fact that the lattice coupling element can twist the one-turn map and manipulate its eigenvectors, enabling control of the weights for the projection of the three eigenemittances into different physical dimensions. It can be anticipated that many interesting issues related to the beam dynamics will arise in the case of a general 3D coupled lattice. A study on this topic is ongoing and will be reported elsewhere in the future.

\section{NONLINEAR TRANSVERSE-LONGITUDINAL COUPLING}

\section{A. Dependence of the average path length on the betatron oscillation amplitudes}

After investigating linear transverse-longitudinal coupling, we will now examine the case of nonlinear coupling. However, we consider only second-order effects here. A general discussion of the nonlinear dynamics is beyond the scope of this paper. The second-order transverselongitudinal coupling considered here originates from a dependence of the average path length on the betatron oscillation amplitudes, which can be expressed by a concise formula

$$
\Delta C=-2 \pi\left(\xi_{x} J_{x}+\xi_{y} J_{y}\right),
$$

where $\Delta C$ is the average path-length deviation relative to the ideal particle, and $\xi_{x, y}$ and $J_{x, y}$ are the horizontal (vertical) chromaticity and betatron invariant, respectively. This simple relation is a result of the symplecticity of the Hamiltonian dynamics, as pointed out by Forest [56] and other authors $[57,58]$. It is called a second-order coupling because the betatron invariant is a second-order term with respect to the transverse position and angle. Note that Eq. (52) is accurate only for the cases of multiple passes or multiple betatron oscillations as it is a betatron-phase-averaged result. For the case of a single pass with only a few betatron oscillations, there will be an extra term, depending on the betatron phase advance, in the path length formula.

This effect has previously been theoretically analyzed by several authors in different contexts [56-61]. Due to this effect, particles with different betatron amplitudes lose synchronization with each other when traversing a lattice with nonzero chromaticity. This leads to a stringent requirement on the beam emittance for FELs in the x-ray regime (XFELs), as microbunching can be smeared out by this effect when the beam is traveling through the undulator [62]. This effect is also crucial in nonscaling fixed-field alternating-gradient (FFAG) accelerators for muon acceleration [58], as a muon beam typically has a large emittance. Furthermore, the natural chromaticities of a 
linear nonscaling FFAG accelerator are usually not corrected to achieve a large transverse acceptance. This effect may also have an impact on the momentum and dynamic aperture in a storage ring $[63,64]$, for example, due to the Touschek scattering-induced large betatron amplitude or the large natural chromaticity in a low-emittance lattice. It is also relevant in other applications demanding particle synchronization. In this paper, we wish to investigate and emphasize the importance of this nonlinear transverselongitudinal coupling in storage-ring-based schemes such as SSMB.

\section{B. Oscillation center shift, energy widening, bunch lengthening and distortion from a Gaussian distribution}

As mentioned, the second-order transverse-longitudinal coupling effect can smear out microbunching in XFELs. Methods of overcoming this influence are referred to as "beam conditioning". Several such methods have been proposed since the first publication of Sessler et al. [62]. The basic idea of these proposals is to compensate for the difference in path length through a difference in velocity by establishing a correlation between the betatron amplitude and the particle energy. In a storage ring, unlike in a singlepass device, the rf cavity will "condition" the beam automatically, causing all particles to synchronize with it in an average sense through phase stabilization (bunching). This is accomplished by introducing a betatron-amplitudedependent energy shift to compensate for the path-length difference arising from the betatron oscillations,

$$
\Delta \delta=-\frac{\Delta C}{\alpha C_{0}}
$$

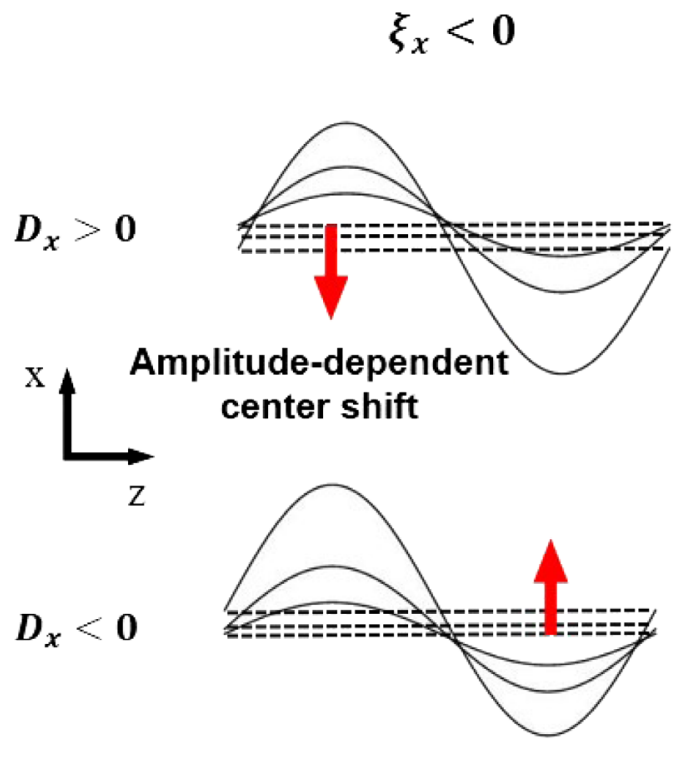

where $\alpha$ is the momentum compaction factor of the ring. This shift will result in the beam energy widening in a quasi-isochronous ring with nonzero chromaticity, because different particles have different betatron invariants, as theoretically analyzed by Shoji [61]. This widening will become more significant with decreasing momentum compaction.

Due to the energy shift, there will also be an amplitudedependent shift in the betatron oscillation center at dispersive locations. The shift direction is determined by the signs of $\alpha, \xi_{x, y}$, and $D_{x, y}$, and the magnitude of the shift is determined by the magnitudes of $J_{x, y}, \alpha, \xi_{x, y}$, and $D_{x, y}$. The physical pictures of the betatron center shift resulting from this effect are shown in Fig. 8.

When quantum excitation is also taken into account, the total relative energy deviation of a particle with respect to the ideal particle is

$$
\delta=\Delta \delta+\delta_{\mathrm{qe}}
$$

where $\delta_{\text {qe }}$ represents the quantum excitation contribution. Finding a general analytical formula for the steady-state distribution of the particles is a complex task and, at the same time, not very useful. Simpler expressions can be obtained based on reasonable approximations. Since the vertical emittance is usually much smaller than the horizontal emittance in a planar ring, here, we consider only the contribution from the horizontal emittance. When the coupling is not very strong, the distributions of $J_{x}$ and $\delta_{\text {qe }}$ are still approximately exponential and Gaussian, respectively, and are independent of each other,
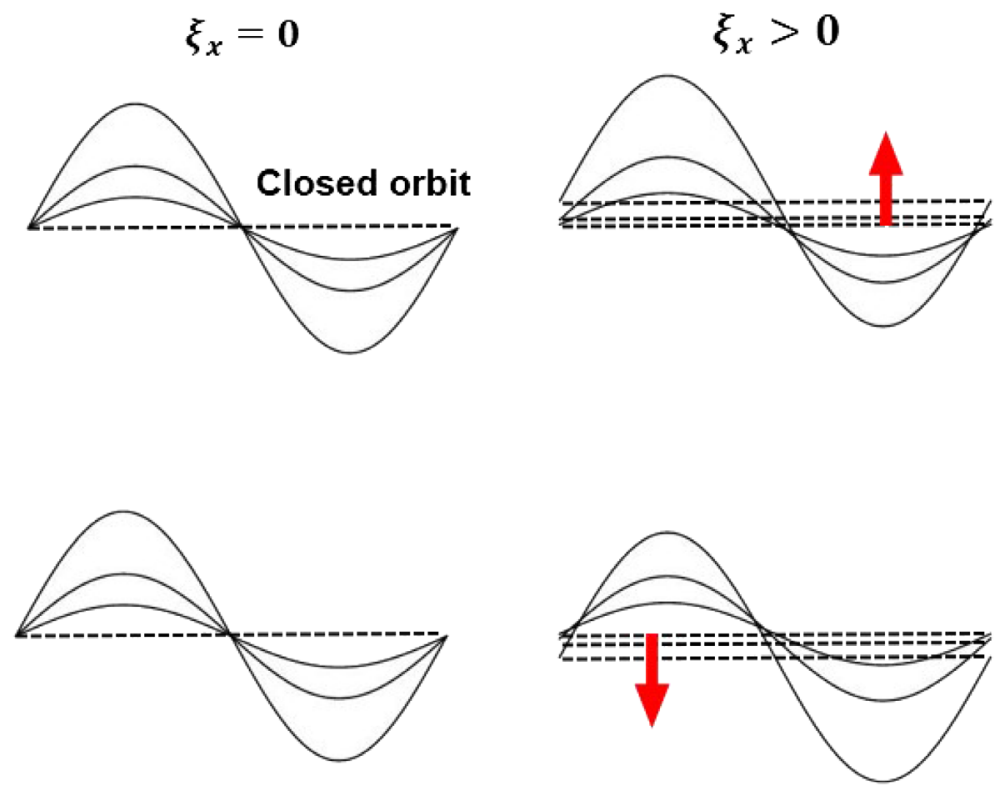

FIG. 8. Physical pictures of the amplitude-dependent shift of betatron oscillation center in the case of a positive momentum compaction. Only a horizontal betatron oscillation is considered in this illustration. 
$\psi\left(J_{x}\right)=\frac{1}{2 \pi \epsilon_{x 0}} e^{-\frac{J_{x}}{\epsilon_{x 0}}}, \quad \psi\left(\delta_{\mathrm{qe}}\right)=\frac{1}{\sqrt{2 \pi} \sigma_{\delta 0}} e^{-\frac{\delta_{\mathrm{qe}}^{2}}{2 \sigma_{\delta 0}^{2}}}$,

where $\epsilon_{x 0}$ and $\sigma_{\delta 0}$ are the natural horizontal emittance and energy spread. The distribution of $\delta$ is thus an exponentially modified Gaussian because it is the sum of an exponential and a normal random variable,

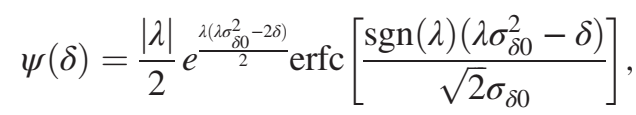

where $\lambda=\frac{\alpha C_{0}}{2 \pi \xi_{x} \epsilon_{x 0}}, \operatorname{sgn}(\mathrm{x})$ is the sign function and $\operatorname{erfc}(\mathrm{x})$ is the complementary error function, defined as $\operatorname{erfc}(x)=1-$ $\operatorname{erf}(x)=\frac{2}{\sqrt{\pi}} \int_{x}^{\infty} e^{-t^{2}} d t$. The direction of long non-Gaussian tail of the energy distribution is determined by the signs of $\alpha$ and $\xi_{x}$.

Because of the dispersion and dispersion angle, the nonGaussian particle energy distribution can also be reflected in the transverse dimension. When this nonlinear coupling is considered, the horizontal position and angle of a particle in the storage ring are
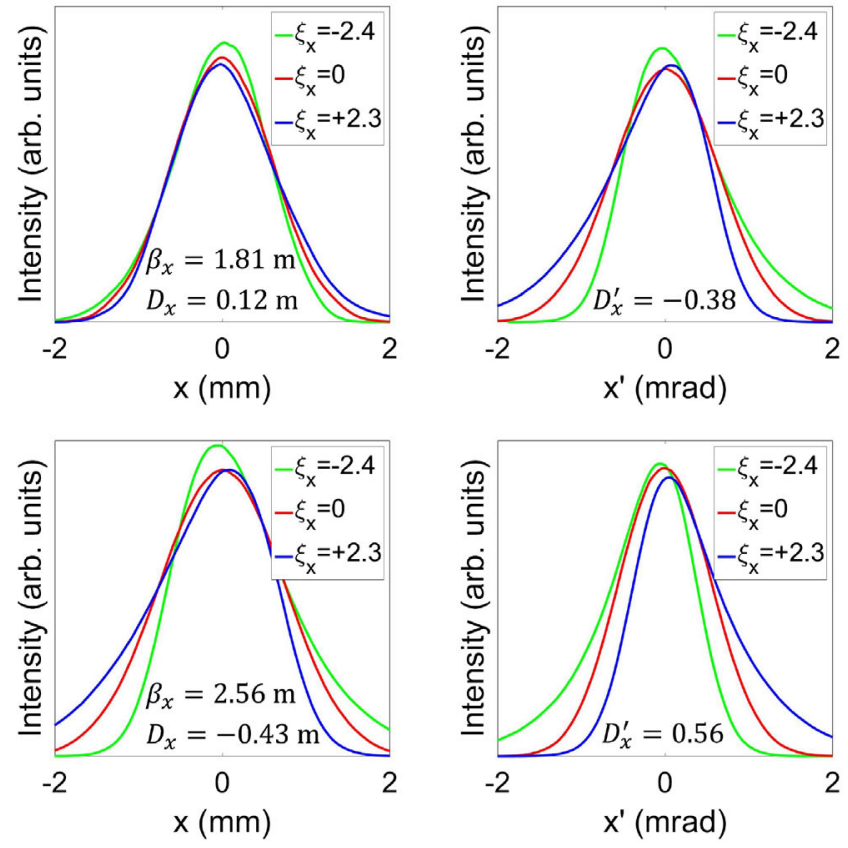
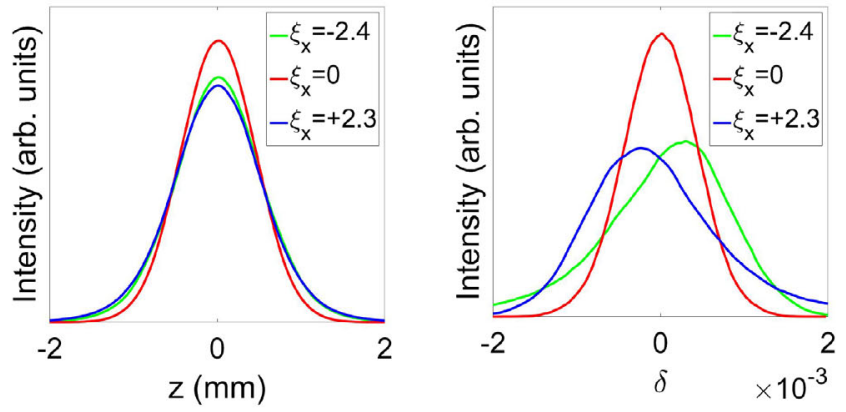

$$
\begin{aligned}
& x=\sqrt{2 J_{x} \beta_{x}} \cos \psi_{x}+D_{x}\left(\delta_{\mathrm{qe}}+\frac{2 \pi \xi_{x} J_{x}}{\alpha C_{0}}\right) \\
& x^{\prime}=-\sqrt{2 J_{x} / \beta_{x}}\left(\alpha_{x} \cos \psi_{x}+\sin \psi_{x}\right)+D_{x}^{\prime}\left(\delta_{\mathrm{qe}}+\frac{2 \pi \xi_{x} J_{x}}{\alpha C_{0}}\right) .
\end{aligned}
$$

It is assumed that the concept of the Courant-Snyder functions is still approximately valid in Eq. (57).

With these approximations, the variance of $\delta$ is

$$
\sigma_{\delta}^{2}=\sigma_{\delta 0}^{2}+\left(\frac{2 \pi \epsilon_{x 0} \xi_{x}}{\alpha C_{0}}\right)^{2} .
$$

By assuming the MLS parameters shown in Table I and applying $\alpha=1 \times 10^{-4}$ and $\xi_{x}=2$, one can find that the energy spread contributed by this effect can be as significant as its natural value.

As discussed in [61], a shift in the energy center corresponds to a shift in the synchronous rf phase $\phi_{s}$,

$$
\Delta \phi_{s} \approx J_{\epsilon} \tan \phi_{s} \Delta \delta
$$

where $J_{\epsilon}$ is the longitudinal damping partition number. Therefore, particles with different betatron amplitudes will oscillate around different fixed points in the longitudinal dimension, thus lengthening the bunch. The change in the
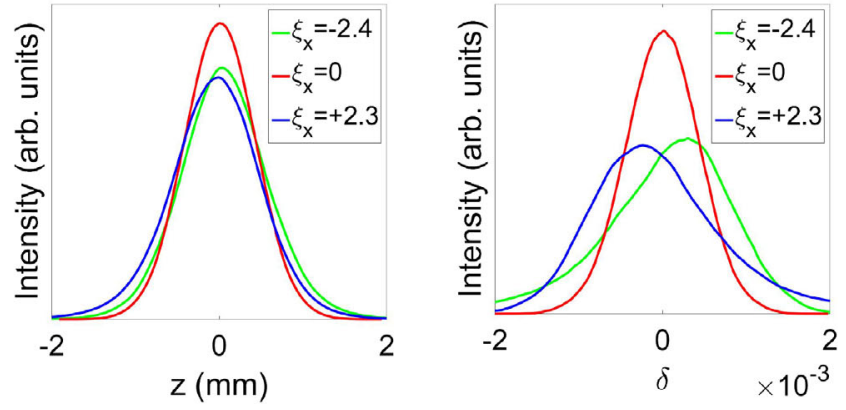

FIG. 9. Energy widening, bunch lengthening and distortion from a Gaussian distribution induced by a nonvanishing horizontal chromaticity. From top to bottom, the particle tracking results for distributions of $x, x^{\prime}, z$ and $\delta$ are shown at two dispersive locations in the MLS under three different horizontal chromaticities $\xi_{x}$. The direction of the long non-Gaussian tail for $\delta$ is related to the signs of $\alpha$ and $\xi_{x}$, while for $x$ and $x^{\prime}$ they are also dependent on $D_{x}$ and $D_{x}^{\prime}$, respectively. The simulation was conducted using the ELEGANT [31] code with a beam energy of $630 \mathrm{MeV}$, an rf voltage of $500 \mathrm{kV}$ and the application of $\alpha=1 \times 10^{-4}$. In each simulation, eight particles were tracked for $5 \times 10^{6}$ turns, corresponding to approximately 73 longitudinal damping times. 
synchronous rf phase in a unit of the longitudinal coordinate, $\Delta z_{s}$, is related to the relative change in energy, $\Delta \delta$, according to

$$
\frac{\Delta z_{s}}{\sigma_{z 0}}=\frac{\nu_{s} J_{\epsilon} \tan \phi_{s}}{h|\alpha|} \frac{\Delta \delta}{\sigma_{\delta 0}} \propto \frac{1}{\sqrt{|\alpha|}} \frac{\Delta \delta}{\sigma_{\delta 0}},
$$

where $\nu_{s}$ is the synchrotron tune and $\sigma_{z 0}$ and $\sigma_{\delta 0}$ are the natural bunch length and energy spread, respectively.

The critical value of alpha, $\alpha_{c}$, when the relative change of bunch length and energy spread are the same can be calculated to be

$$
\frac{\nu_{s} J_{\epsilon} \tan \phi_{s}}{h\left|\alpha_{c}\right|}=1 \Rightarrow\left|\alpha_{c}\right|=\frac{J_{\epsilon} T_{0} \tan \phi_{s}}{\pi h \tau_{\delta}},
$$

where $\tau_{\delta}=1 / k_{s}$ is the longitudinal damping time and $h$ is the harmonic number of the rf cavity (or, for SSMB, the laser modulator). As an example, we use the MLS parameters given in Table I and consider the application of an $\mathrm{rf}$ voltage of $500 \mathrm{kV}$, which corresponds to a synchronous rf phase of $\phi_{s}=0.018 \mathrm{rad}$. The critical value of alpha is then $\left|\alpha_{c}\right| \approx 2.1 \times 10^{-9}$, which is more than four orders of magnitude smaller than the standard low alpha value at the MLS. Therefore, the relative bunch lengthening resulting from this effect is much less significant than the corresponding energy widening at the MLS.

\section{Simulation}

Several particle tracking simulations were conducted using the MLS lattice with the parameters presented in Table I to confirm the analysis. Two dispersive locations, with different signs and magnitudes of $D_{x}$, were selected as the observation points in the simulations. The simulation results are shown in Fig. 9. The energy widening and distortion from Gaussian behaviors are as expected and, indeed, are more significant than the bunch lengthening when $\alpha=1 \times 10^{-4}$. At the two observation points, widening and distortion of the particle energy distribution also manifest in the transverse dimension through $D_{x}$ and $D_{x}^{\prime}$. The related optic functions at the two observation points are also shown in the profiles of $x$ and $x^{\prime}$. Note that the directions of the long non-Gaussian tails of the profiles and their relation to the signs of $\xi_{x}, D_{x}$, and $D_{x}^{\prime}$. We conclude that the simulation results agree well with the analysis and physical pictures presented above.

\section{Discussion}

Two experiments $[65,66]$ were conducted to verify the relation in Eq. (52) and the amplitude-dependent shift of the betatron oscillation center. In particular, the influence of this effect on the equilibrium beam characteristics in an electron storage ring, as analyzed in [61] and this paper, were recently experimentally investigated at the MLS.
The quantitative measurements and their good agreement with the theoretical calculations confirm that this effect broadens the energy spread and distorts the beam from a Gaussian shape in both the horizontal and longitudinal dimensions in a low-alpha ring with nonzero horizontal chromaticity. Further details on the analysis of this effect and the experimental results can be found in [67].

For SSMB, this effect will have both a transient impact on microbunch transportation, similar to that in an XFEL, and a steady-state influence on the beam characteristics, as analyzed in [61] and this paper. While energy widening and distortion seem to be undesirable for some applications, they could actually be beneficial in some cases because these effects can help to stabilize many collective instabilities. In most cases, however, the bunch lengthening is much less notable than the energy widening. In addition, for SSMB, because the harmonic number $h$ is much larger than that of a conventional ring, it can be seen from Eqs. (60) and (61) that compared to the energy widening, the steady-state bunch lengthening in this case is negligible. Therefore, in the case of low-alpha rings and certain SSMB scenarios, it may actually be possible to boost the stable coherent radiation power by making use of this effect. For example, it has been demonstrated at the MLS that the stable single-bunch current can be enhanced by more than one order of magnitude by increasing the absolute value of the horizontal chromaticity from 0 to a value larger than 3 , with the head-tail effect and other collective effects, such as the longitudinal microwave instability, adequately suppressed. The increase in the $\mathrm{THz}$ power due to a higher stable beam current turns out to overcompensate for the decrease due to the slight bunch lengthening of this effect. Therefore, this approach is now implemented at the MLS as the standard low-alpha mode for Fourier-transform infrared spectroscopy (FTIR) applications [68].

\section{SUMMARY}

In this paper, the basic concept and promising potential of SSMB are briefly reviewed along with recent progress in this area. The related single-particle microbunching dynamics are theoretically and numerically studied, and importance results are presented. For the storage of microbunches in microbuckets, the longitudinal quantum excitation due to stochastic photon emission is a fundamental effect that first requires careful treatment. Although it typically causes bunch lengthening, linear transverselongitudinal coupling can nevertheless be favorable for bunch compression and harmonic generation when the transverse emittance is small because of the flexibility with which the three eigenemittances can be projected into different physical dimensions. On the other hand, nonlinear transverse-longitudinal coupling may transiently degrade the microbunching and steadily distort the beam. However, the energy widening and beam distortion resulting from such nonlinear coupling can also be helpful in stabilizing 
collective instabilities and thus may boost the stable coherent radiation power. The possibility of using nonlinear momentum compaction for high harmonic bunching is also proposed. We believe that the investigations presented in this paper will be useful for many applications in accelerator physics involving the precise phase-space manipulations of beams.

\section{ACKNOWLEDGMENTS}

The authors thank T. Rui, G. Xu, W. Wan, X. Huang, Y. Jiao, D. Wang, C. Feng, B. Jiang, A. Matveenko, Y. Petenev, J. Li, L. Yan, Y. Zhang, Z. Pan, J. Wu, and other colleagues for helpful discussions. X. J. Deng wishes to express special appreciation to T. Rui for the help at the beginning of his study of accelerator physics. X. J. Deng is also thankful for the help and hospitality extended to him by the Helmholtz-Zentrum Berlin and PTB colleagues, especially $\mathrm{J}$. Li, during his visits to the MLS. This work is supported by the Tsinghua University Initiative Scientific Research Program No. 20191081195, China.

[1] J. M. Madey, Stimulated emission of bremsstrahlung in a periodic magnetic field, J. Appl. Phys. 42, 1906 (1971).

[2] D. A. G. Deacon, L. R. Elias, J. M. J. Madey, G. J. Ramian, H. A. Schwettman, and T. I. Smith, First Operation of a Free-Electron Laser, Phys. Rev. Lett. 38, 892 (1977).

[3] P. Emma, R. Akre, J. Arthur, R. Bionta, C. Bostedt, J. Bozek, A. Brachmann, P. Bucksbaum, R. Coffee, F.-J. Decker et al., First lasing and operation of an ångstromwavelength free-electron laser, Nat. Photonics 4, 641 (2010).

[4] B. Girard, Y. Lapierre, J. M. Ortega, C. Bazin, M. Billardon, P. Elleaume, M. Bergher, M. Velghe, and Y. Petroff, Optical Frequency Multiplication by an Optical Klystron, Phys. Rev. Lett. 53, 2405 (1984).

[5] M. Labat, M. Hosaka, A. Mochihashi, M. Shimada, M. Katoh, G. Lambert, T. Hara, Y. Takashima, and M. Couprie, Coherent harmonic generation on UVSOR-II storage ring, Eur. Phys. J. D 44, 187 (2007).

[6] G. De Ninno, E. Allaria, M. Coreno, F. Curbis, M. B. Danailov, E. Karantzoulis, A. Locatelli, T. O. Menteş, M. A. Nino, C. Spezzani, and M. Trovò, Generation of Ultrashort Coherent Vacuum Ultraviolet Pulses Using Electron Storage Rings: A New Bright Light Source for Experiments, Phys. Rev. Lett. 101, 053902 (2008).

[7] A. A. Zholents and M. S. Zolotorev, Femtosecond X-Ray Pulses of Synchrotron Radiation, Phys. Rev. Lett. 76, 912 (1996).

[8] R. W. Schoenlein, S. Chattopadhyay, H. H. W. Chong, T. E. Glover, P. A. Heimann, C. V. Shank, A. A. Zholents, and M. S. Zolotorev, Generation of femtosecond pulses of synchrotron radiation, Science 287, 2237 (2000).

[9] S. Khan, K. Holldack, T. Kachel, R. Mitzner, and T. Quast, Femtosecond Undulator Radiation from Sliced Electron Bunches, Phys. Rev. Lett. 97, 074801 (2006).
[10] D. F. Ratner and A. W. Chao, Steady-State Microbunching in a Storage Ring for Generating Coherent Radiation, Phys. Rev. Lett. 105, 154801 (2010).

[11] A. Chao, E. Granados, X. Huang, D. Ratner, and H.-W. Luo, High power radiation sources using the steadystate microbunching mechanism, in Proceedings of the 7th International Particle Accelerator Conference (IPAC'16), Busan, Korea, 2016 (JACoW, Geneva, 2016), pp. 1048-1053, https://accelconf.web.cern.ch/ ipac2016/papers/tuxb01.pdf.

[12] C. Tang, X. Deng, A. Chao, W. Huang, T. Rui, J. Feikes, J. Li, M. Ries, A. Hoehl, D. Ratner et al., An overview of the progress on SSMB, in Proceedings of the 60th ICFA Advanced Beam Dynamics Workshop on Future Light Sources (FLS'18), Shanghai, China, 2018 (JACoW, Geneva, 2018), pp. 166-170, http://accelconf.web.cern .ch/fls2018/papers/thp2wb02.pdf.

[13] G. Wüstefeld, A. Jankowiak, J. Knobloch, and M. Ries, Simultaneous long and short electron bunches in the BESSY II storage ring, in Proceedings of the 2th International Particle Accelerator Conference (IPAC'11), San Sebastián, Spain, 2011 (EPS-AG, Spain, 2011), pp. 2936-2938, https://accelconf.web.cern.ch/ipac2011/ papers/thpc014.pdf.

[14] P. Goslawski, M. Ries, M. Ruprecht, and G. Wüstefeld, The low-alpha lattice and bunch length limits at BESSYVSR, in Proceedings of the 5th International Particle Accelerator Conference (IPAC'14), Dresden, Germany, 2014 (JACoW, Geneva, 2014), pp. 216-218, https:// accelconf.web.cern.ch/ipac2014/papers/mopro058.pdf.

[15] P. Schnizer, M. Ries, W. Anders, Y. Bergmann, H. Ehmler, P. Goslawski, A. Jankowiak, J. Knobloch, A. Neumann, K. Ott et al., Status of the BESSY VSR project, in Proceedings of the 9th International Particle Accelerator Conference (IPAC'18), Vancouver, BC, Canada, 2018 (JACoW, Geneva, 2018), pp. 4138-4141, https:// accelconf.web.cern.ch/ipac2018/papers/thpmf038.pdf.

[16] A. Chao, X. Deng, W. Huang, T. Rui, C. Tang, J. Feikes, R. Klein, J. Li, M. Ries, A. Hoehl et al., A compact high-power radiation source based on steady-state microbunching mechanism, SLAC Technical Report No. SLACPUB-17241, 2018, https://www.slac.stanford.edu/pubs/ slacpubs/17000/slac-pub-17241.pdf.

[17] X. Deng, A. Chao, W. Huang, T. Rui, C. Tang, J. Feikes, M. Ries, R. Klein, and D. Ratner, From coherent harmonic generation to steady state microbunching, in Proceedings of the 9th International Particle Accelerator Conference (IPAC'18), Vancouver, BC, Canada, 2018 (JACoW, Geneva, 2018), pp. 4583-4586, https://accelconf.web .cern.ch/ipac2018/papers/thpmk113.pdf.

[18] T. Rui, X. Deng, A. Chao, W. Huang, and C. Tang, Strong focusing lattice design for SSMB, in Proceedings of the 60th ICFA Advanced Beam Dynamics Workshop on Future Light Sources (FLS'18), Shanghai, China, 2018 (JACoW, Geneva, 2018), pp. 113-116, https://accelconf.web.cern .ch/fls2018/papers/wep2pt014.pdf.

[19] Z. Pan, T. Rui, W. Wan, A. Chao, X. Deng, Y. Zhang, W. Huang, and C. Tang, A storage ring design for steady-state microbunching to generate coherent EUV light source, in Proceedings of the 39th International Free Electron Laser 
Conference (FEL'19), Hamburg, Germany, 2019 (JACoW, Geneva, 2019), pp. 700-703, https://accelconf.web.cern .ch/AccelConf/fel2019/papers/thp055.pdf.

[20] C. Li, C. Feng, B. Jiang, and A. Chao, Lattice design for the reversible SSMB, in Proceedings of the 10th International Particle Accelerator Conference (IPAC'19), Melbourne, Australia, 2019 (JACoW, Geneva, 2019), pp. 1507-1509, https://accelconf.web.cern.ch/ipac2019/ papers/tupgw045.pdf.

[21] Y. Jiao, D. F. Ratner, and A. W. Chao, Terahertz coherent radiation from steady-state microbunching in storage rings with $X$-band radio-frequency system, Phys. Rev. Accel. Beams 14, 110702 (2011).

[22] D. Ratner and A. Chao, Reversible seeding in storage rings, in Proceedings of the 33th International Free Electron Laser Conference (FEL'11), Shanghai, China, 2011, pp. 57-60, https://accelconf.web.cern.ch/FEL2011/ papers/mopb23.pdf.

[23] M. Sands, The physics of electron storage rings: An introduction, SLAC Technical Report No. SLAC-121, 1970, https://www.slac.stanford.edu/pubs/slacreports/ reports02/slac-r-121.pdf.

[24] Y. Shoji, H. Tanaka, M. Takao, and K. Soutome, Longitudinal radiation excitation in an electron storage ring, Phys. Rev. E 54, R4556 (1996).

[25] A. W. Chao and M. J. Lee, Particle distribution parameters in an electron storage ring, J. Appl. Phys. 47, 4453 (1976).

[26] B. Nash, Analytical approach to eigen-emittance in storage rings, Ph.D. thesis, Stanford University, Stanford, California, USA, 2006.

[27] A. W. Chao, Evaluation of beam distribution parameters in an electron storage ring, J. Appl. Phys. 50, 595 (1979).

[28] K. Ohmi, K. Hirata, and K. Oide, From the beam-envelope matrix to synchrotron-radiation integrals, Phys. Rev. E 49, 751 (1994).

[29] J. Feikes, M. von Hartrott, M. Ries, P. Schmid, G. Wüstefeld, A. Hoehl, R. Klein, R. Müller, and G. Ulm, Metrology Light Source: The first electron storage ring optimized for generating coherent $\mathrm{THz}$ radiation, Phys. Rev. Accel. Beams 14, 030705 (2011).

[30] M. Ries, Nonlinear Momentum Compaction and Coherent Synchrotron Radiation at the Metrology Light Source, Dissertation, Humboldt University of Berlin, Berlin, Germany, 2014.

[31] M. Borland, ELEGANT: A flexible SDDS-compliant code for accelerator simulation, ANL Advanced Photon Source Report No. LS-287, 2000, https://publications.anl.gov/ anlpubs/2000/08/36940.pdf.

[32] C. Teng, Minimizing the emittance in designing the lattice of an electron storage ring, FNAL Technical Report No. Fermilab-TM-1269, 1984.

[33] G. Xu and W. Wan (private communication).

[34] C. Pellegrini and D. Robin, Quasi-isochronous storage ring, Nucl. Instrum. Methods Phys. Res., Sect. A 301, 27 (1991).

[35] L. H. Yu, Generation of intense uv radiation by subharmonically seeded single-pass free-electron lasers, Phys. Rev. A 44, 5178 (1991).

[36] L.-H. Yu, M. Babzien, I. Ben-Zvi, L. DiMauro, A. Doyuran, W. Graves, E. Johnson, S. Krinsky, R. Malone,
I. Pogorelsky et al., High-Gain Harmonic-Generation FreeElectron Laser, Science 289, 932 (2000).

[37] G. Stupakov, Using the Beam-Echo Effect for Generation of Short-Wavelength Radiation, Phys. Rev. Lett. 102, 074801 (2009).

[38] D. Xiang and G. Stupakov, Echo-enabled harmonic generation free electron laser, Phys. Rev. Accel. Beams 12, 030702 (2009).

[39] G. Stupakov and M. Zolotorev, Using laser harmonics to increase bunching factor in EEHG, in Proceedings of the 33th International Free Electron Laser Conference (FEL'11), Shanghai, China, 2011, pp. 45-48, https:// accelconf.web.cern.ch/FEL2011/papers/mopb19.pdf.

[40] D. Ratner and A. Chao, Seeded radiation sources with sawtooth waveforms, in Proceedings of the 33th International Free Electron Laser Conference (FEL'11), Shanghai, China, 2011, pp. 53-56, https://accelconf.web .cern.ch/FEL2011/papers/mopb21.pdf.

[41] X. Deng, Y. Zhang, A. Chao, W. Huang, and C. Tang, Transverse-longitudinal coupling for harmonic generation and bunch length manipulation, in Proceedings of the 10th International Particle Accelerator Conference (IPAC'19), Melbourne, Australia, 2019 (JACoW, Geneva, 2019), pp. 3160-3163, https://accelconf.web.cern.ch/ipac2019/ papers/wepts028.pdf.

[42] Y. Shoji, Bunch lengthening by a betatron motion in quasiisochronous storage rings, Phys. Rev. Accel. Beams 7, 090703 (2004).

[43] G. Wüstefeld, Horizontal-longitudinal coupling in the FEL beam line, BESSY Tech. Report No. BESSY TB 227/05, 2005.

[44] X. Huang, Matrix formalism of synchrobetatron coupling, Phys. Rev. Accel. Beams 10, 014002 (2007).

[45] M. Shimada, M. Katoh, M. Adachi, T. Tanikawa, S. Kimura, M. Hosaka, N. Yamamoto, Y. Takashima, and T. Takahashi, Transverse-Longitudinal Coupling Effect in Laser Bunch Slicing, Phys. Rev. Lett. 103, 144802 (2009).

[46] Y. Shoji, Transient bunch lengthening by a betatron motion along bending sections, Nucl. Instrum. Methods Phys. Res., Sect. A 637, S120 (2011).

[47] E. D. Courant and H.S. Snyder, Theory of the alternating-gradient synchrotron, Ann. Phys. (N.Y.) 3, 1 (1958).

[48] A. W. Chao, K. H. Mess, M. Tigner, and F. Zimmermann, Handbook of Accelerator Physics and Engineering, 2nd ed. (World Scientific, Singapore, 2013), pp. 65-66.

[49] J. P. MacArthur, A. A. Lutman, J. Krzywinski, and Z. Huang, Microbunch Rotation and Coherent Undulator Radiation from a Kicked Electron Beam, Phys. Rev. X 8, 041036 (2018).

[50] A. J. Dragt, F. Neri, and G. Rangarajan, General moment invariants for linear Hamiltonian systems, Phys. Rev. A 45, 2572 (1992).

[51] M. Cornacchia and P. Emma, Transverse to longitudinal emittance exchange, Phys. Rev. Accel. Beams 5, 084001 (2002).

[52] P. Emma, Z. Huang, K.-J. Kim, and P. Piot, Transverse-tolongitudinal emittance exchange to improve performance of high-gain free-electron lasers, Phys. Rev. Accel. Beams 9, 100702 (2006). 
[53] H. Deng and C. Feng, Using Off-Resonance Laser Modulation for Beam-Energy-Spread Cooling in Generation of Short-Wavelength Radiation, Phys. Rev. Lett. 111, 084801 (2013).

[54] C. Feng, H. Deng, D. Wang, and Z. Zhao, Phase-merging enhanced harmonic generation free-electron laser, New J. Phys. 16, 043021 (2014).

[55] C. Feng and Z. Zhao, A storage ring based free-electron laser for generating ultrashort coherent EUV and X-ray radiation, Sci. Rep. 7, 4724 (2017).

[56] É. Forest, Beam Dynamics: A New Attitude and Framework (Harwood Academic Publisher, Amsterdam, 1998), Chap. 9, p. 264.

[57] A. Chao, Lecture notes on topics in accelerator physics, SLAC Tech. Report No. SLAC-PUB-9574, 2002, https:// www.slac.stanford.edu/ achao/lecturenotes.html.

[58] J. S. Berg, Amplitude dependence of time of flight and its connection to chromaticity, Nucl. Instrum. Methods Phys. Res., Sect. A 570, 15 (2007).

[59] A. Artamonov, I. S. Derbenev, and N. Inozemtsev, The possibility of controlling the dispersion characteristics of particle motion in an undulator, Zh. Tekh. Fiz. 59, 214 (1989).

[60] L. Emery, Coupling of betatron motion to the longitudinal plane through path lengthening in low alpha(c) storage rings, in Proceedings of the 15th International Conference on High Energy Accelerators, edited by J. Rossbach (World Scientific, Singapore, 1993), pp. 1172-1174.

[61] Y. Shoji, Dependence of average path length betatron motion in a storage ring, Phys. Rev. Accel. Beams 8, 094001 (2005).
[62] A. M. Sessler, D. H. Whittum, and L.-H. Yu, Radiofrequency beam conditioner for fast-wave free-electron generators of coherent radiation, Phys. Rev. Lett. 68, 309 (1992).

[63] M. Takao, Impact of betatron motion on path lengthening and momentum aperture in a storage ring, in Proceedings of the 11th European Particle Accelerator Conference (EPAC'08), Genoa, Italy, 2008, pp. 3152-3154, http:// accelconf.web.cern.ch/accelconf/e08/papers/thpc072.pdf.

[64] L. Hoummi, J. R. Lopez, C. Welsch, A. Loulergue, and R. Nagaoka, Beam dynamics in MBA lattices with different chromaticity correction schemes, in Proceedings of the 10th International Particle Accelerator Conference (IPAC'19), Melbourne, Australia, 2019 (JACoW, Geneva, 2019), pp. 346-349, https://accelconf .web.cern.ch/ipac2019/papers/mopgw096.pdf.

[65] Y. Shoji, M. Takao, and T. Nakamura, Amplitude dependent shift of betatron oscillation center, Phys. Rev. Accel. Beams 17, 064001 (2014).

[66] S. Machida, D. J. Kelliher, C. S. Edmonds, I. W. Kirkman, J. S. Berg, J. K. Jones, B. D. Muratori, and J. M. Garland, Amplitude-dependent orbital period in alternating gradient accelerators, Prog. Theor. Exp. Phys. 2016, 033G01 (2016).

[67] X. J. Deng, R. Klein, A. W. Chao, A. Hoehl, W. H. Huang, J. Li, J. Lubeck, Y. Petenev, M. Ries, I. Seiler et al., Widening and distortion of the particle energy distribution by chromaticity in quasi-isochronous rings, Phys. Rev. Accel. Beams 23, 044001 (2020).

[68] J. Feikes and A. Hoehl, (private communication). 